Purdue University

Purdue e-Pubs

2010

\title{
Electrical Actuation-Induced Droplet Transport on Smooth and Superhydrophobic Surfaces
}

\author{
V. Bahadur \\ Purdue University - Main Campus \\ S V. Garimella \\ Purdue University, sureshg@purdue.edu
}

Follow this and additional works at: http://docs.lib.purdue.edu/coolingpubs

Bahadur, V. and Garimella, S V., "Electrical Actuation-Induced Droplet Transport on Smooth and Superhydrophobic Surfaces" (2010). CTRC Research Publications. Paper 145.

http://docs.lib.purdue.edu/coolingpubs/145

This document has been made available through Purdue e-Pubs, a service of the Purdue University Libraries. Please contact epubs@purdue.edu for additional information. 
Electrical actuation-induced droplet transport on smooth and superhydrophobic surfaces by

Vaibhav Bahadur and Suresh V. Garimella reprinted from

\section{International Journal of Micro-Nano} scale Transport Volume 1 - Number $1 \cdot$ March 2010

Multi-Science Publishing ISSN 1.759-3093 


\title{
Electrical actuation-induced droplet transport on smooth and superhydrophobic surfaces
}

\author{
Vaibhav Bahadur and Suresh V. Garimella ${ }^{1}$ \\ School of Mechanical Engineering and Birck Nanotechnology Center, \\ Purdue University, West Lafayette, Indiana 47907-2088, USA
}

\begin{abstract}
Electrical control of liquid droplet motion and wettability has wide-ranging applications in the field of MEMS, lab-on-a-chip devices and surface engineering, in view of the resulting enhanced flow control opportunities, low power consumption and the absence of mechanical moving parts. This article summarizes recent progress towards understanding of the fundamentals underlying electrical actuation of droplets on smooth and superhydrophobic surfaces. Electrical actuation of liquid droplets with widely differing electrical properties on smooth surfaces is first discussed. Electromechanical considerations are employed to study the actuation force on a generic liquid droplet across the entire spectrum of electrical actuation regimes. The challenges in understanding the fluid flow and dissipation mechanisms associated with a discrete moving droplet are discussed. The role of electrical voltages, interfacial energies and surface morphology in determining droplet states (nonwetting Cassie state and wetting Wenzel state) and triggering state transitions on superhydrophobic surfaces is then mapped out. Critical phenomena associated with droplet transitions on superhydrophobic surfaces (energy barrier for the Cassie-Wenzel transition, lack of spontaneous reversibility of the Cassie-Wenzel transition, robustness of the Cassie state, and the role of the roughness elements) are analyzed. The article also highlights key avenues for future research in the fields of electrical actuation-based microfluidics and superhydrophobic surfaces.
\end{abstract}

\section{INTRODUCTION}

Electrical actuation of liquid droplet motion on smooth and superhydrophobic surfaces has been studied as a tool for controlling microfluidic operations which determine the performance of lab-on-a-chip devices, optical systems, fluidic displays and other MEMS-based fluidic devices. Key advantages of electrical actuation of liquids (in the form of discrete droplets) over mechanical actuation include the absence of moving mechanical parts, opportunities for enhanced and reconfigurable flow control, compatibility with liquids of widely different electrical properties, compatibility with existing fabrication techniques, and ease of integration with a chip-based microfluidic platform. Electrical actuation techniques are also very energy-efficient, typically requiring only microwatts of power per microfluidic operation. Owing to these advantages, electrical actuation-based control has attracted significantly more research interest than competing non-mechanical liquid control technologies such as thermocapillarity [1] (reduction of surface tension by temperature, leading to fluid motion), optoelectrowetting [2] (light-controlled surface tension induced pumping) and vapor bubble-based pumping [3].

Electrical actuation of liquid droplets on smooth surfaces and its potential applications have been reviewed by Mugele and Baret [4], Fair [5] and Teh et al. [6]. It is seen that electrical actuation

${ }^{1}$ Corresponding author: sureshg@purdue.edu 
techniques can be reliably and efficiently used to control microfluidic operations such as moving droplets, generating droplets from a pool of liquid, splitting a droplet, and merging and mixing two droplets. There has been noteworthy progress in understanding various secondary phenomena associated with droplet-based systems which include contact-angle saturation [7], satellite-droplet emission [8], the influence of the electrical double layer [9], and dielectric charge trapping [10]; however, the current understanding of these phenomena is still inadequate and requires further examination. Electrical actuation has been attributed in a majority of the available literature as resulting from a change in the solid-liquid interfacial energy upon the application of an electrical voltage (Lippman's equation [4]). However, Jones [11] conclusively argued that electrical actuation is a purely electromechanical phenomenon. The interfacial energies and contact angles do not determine the actuation force in such systems, although interfacial phenomena do influence the fluid mechanics underlying the microfluidic operations. That said, the conventional approach of treating electrical actuation as a consequence of a reduction in interfacial energy simplifies the modeling and yields the same results as those obtained from intricate electromechanical models. Lippman's equation therefore continues to be used for the analysis of electrical actuation; however, it should be considered merely a tool to simplify the analysis and is not an accurate representation of the underlying physics.

The first part of this article summarizes the physics underlying the electrical actuation of droplets on smooth surfaces. The focus of this paper is on the use of electromechanical models to study droplet actuation; in particular, the scaling analysis developed by Kumari et al. [12] is utilized to delineate three different regimes of electromechanical actuation. The fluid mechanics and dissipative mechanisms influencing droplet motion are also summarized; it is seen that significant research is needed to understand the complex fluid mechanics and nonconservative dissipative mechanisms in such systems. Key experimental results in the available literature are briefly summarized and compared to understand features of droplet actuation in the three electromechanical regimes. It should be noted that only droplet motion is discussed in this article; analysis of other microfluidic operations is beyond the scope of the present work.

The interaction of a liquid droplet with artificially or naturally structured surfaces has been studied with the objective of developing superhydrophobic surfaces for a variety of applications. Key physical phenomena associated with superhydrophobic surfaces and existing and potential applications have been extensively analyzed and summarized in the recent literature [13-15]. Superhydrophobicity on structured surfaces has been studied for more than sixty years; however, recent potential applications in the area of drag-reducing and low-friction coatings, energy systems, heat transfer, biomimetics and lab-on-chip systems have resulted in a surge in research interest in the past decade. Heikenfeld and Dhindsa [16] recently summarized the existing work on the influence of an electrical voltage in controlling droplet states and transitions on superhydrophobic surfaces; electrical actuation is shown to be a powerful tool to control droplet states and transitions on superhydrophobic surfaces.

The second part of this article summarizes the fundamental physics underlying electrical control of droplet states and transitions on superhydrophobic surfaces. Analytical energy-minimization models developed by Bahadur and Garimella [17] are utilized to study the role of an electrical voltage in changing the droplet state from non-wetting (Cassie) to wetting (Wenzel). These energy minimizationbased models quantify the role of interfacial energies, surface morphology and electric fields in influencing the droplet state and morphology on a structured surface. Different aspects of the physics involved in the electrically induced Cassie-Wenzel state transition are discussed by utilizing modeling and key experimental results in the available literature. It is seen that the current understanding of droplet state control on superhydrophobic surfaces is limited; a majority of existing studies have analyzed only the electrically induced Cassie-Wenzel transition. The lack of a spontaneous reverse transition to the Cassie state upon removal of the electrical voltage serves as a major impediment to the development of practical applications discussed in the literature. Significant additional research is needed to fully understand and control phenomena which prevent the reversibility of the wetting transition, and the fluid dynamics during the droplet transitions. 


\section{ELECTRICAL ACTUATION OF DROPLETS ON SMOOTH SURFACES \\ 2.1 Electrical actuation regimes}

A typical microfluidic device for the electrical actuation of droplets is represented schematically in Figure 1 [12]. It consists of two flat plates separated by a known spacing. An array of microfabricated and individually addressable electrodes on the bottom plate is used to actuate the droplet. The top plate has a continuous electrode which serves as the ground electrode. The electrodes on the bottom plate are covered with a dielectric layer; the variation in capacitive energy stored in this dielectric layer provides the motive force for electrical actuation. Additionally, this dielectric layer prevents DC current flow through the droplet and eliminates the possibility of electrolysis and/or chemical reactions. The top and bottom plates are coated with a thin layer of a fluoropolymer to render the surface hydrophobic. The droplet may be actuated in air, or instead immersed in an immiscible medium (oil for water droplets) to eliminate droplet evaporation. The droplet size is chosen to be slightly larger than the underlying actuation electrodes to ensure overlap on multiple electrodes. In such a scenario, the droplet moves on to the actuated electrode when a voltage is applied; by pulsing the voltages suitably along an array of electrodes, the droplet can be made to follow any desired path on the surface. It should be noted that the presence of a grounded top plate ensures complete transition of the droplet to the actuated electrode. Open-droplet actuation (without a top plate) has also been demonstrated by grounding the droplet without the use of a top plate.

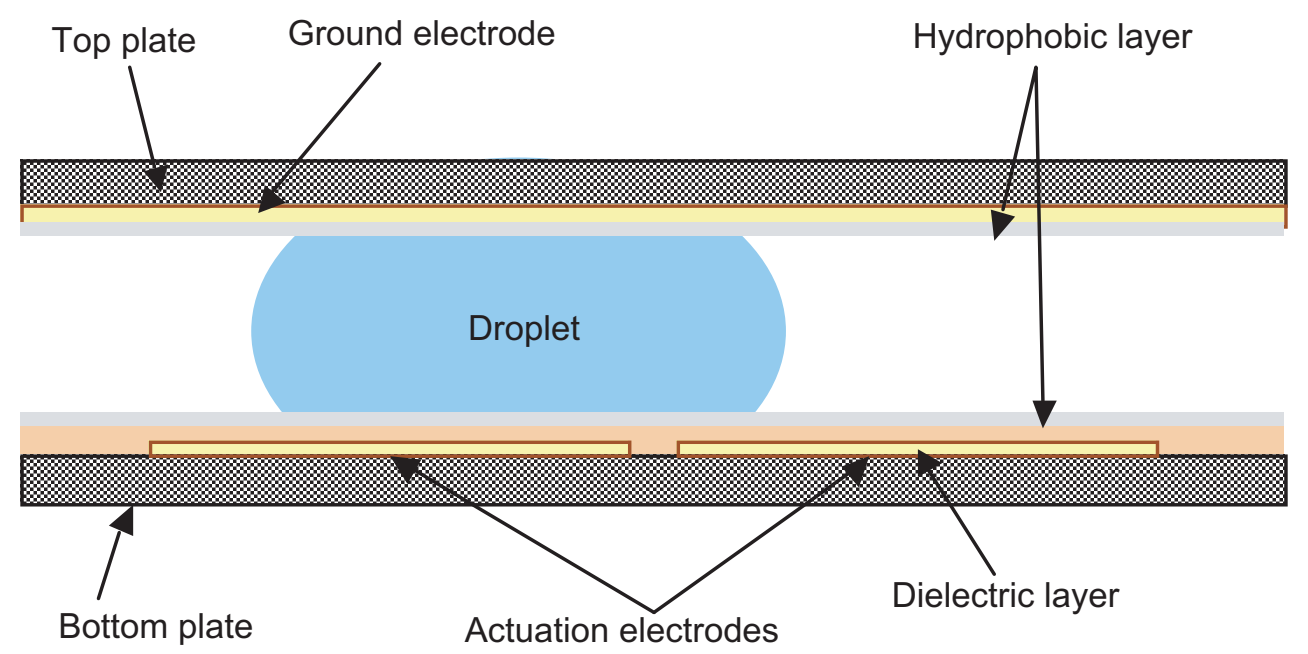

Figure 1. Representative device for electrical actuation of droplets [12].

DC and AC electrical voltages have been used for the actuation of liquids with a wide range of electrical properties. Kumari et al. [12] studied the three different regimes in which a liquid droplet can be electrically actuated: classical electrowetting (EW), AC electrowetting and actuation of dielectric liquids. The electrical properties of the droplet and the underlying dielectric layer along with the frequency of the AC actuation voltage determine the actuation regime. Of the three regimes, classical EW has been studied more extensively than the other two regimes [18-26]. Classical EW systems are based on the premise of the droplet being an electrical conductor and the use of DC voltages for actuation. Water droplets are made electrically conducting by adding a small amount of salt (e.g., potassium chloride). It should be noted that EW systems have been extensively modeled using Lippman's equation [4] which quantifies the reduction in the solid-liquid interfacial energy upon the application of an EW voltage as:

$$
\gamma_{S L}^{V}=\gamma_{S L}^{0}-\frac{1}{2} C_{e q} V^{2}
$$


where $\gamma_{S L}^{V}$ and $\gamma_{S L}^{9}$ are the solid-liquid interfacial energies in the presence and absence respectively of an EW voltage $V$ across the dielectric layer (underlying the droplet) of unit capacitance $C_{e q}$. Lippman's equation implies that the solid-liquid interfacial energy is reduced by the amount of capacitive energy stored in the dielectric layer.

The regime of AC electrowetting [12, 27-34] corresponds to the actuation of an electrically conducting droplet using an AC voltage. Use of AC voltages for electrical actuation is advantageous since the possibility of chemical reactions in the droplet is minimized [29]. Additionally, Berge and Peseux [35] have reported a reduction in contact-angle hysteresis upon the use of AC voltages; this implies a reduction in the threshold voltage for actuation.

The third regime of actuation corresponds to the use of AC or DC voltages for actuating dielectric liquids $[23,36]$; this regime has received much less research attention than the two regimes discussed above. It is important to note that researchers have studied electrically induced wetting [37] and electrically induced capillarity [38,39] of dielectric liquids; however, the present discussion is focused specifically on the actuation of discrete droplets. It should also be noted that the use of dielectric liquids is preferred over electrically conducting liquids in many applications due to the benign and noncorrosive nature of dielectric liquids.

\subsection{Electromechanical modeling of the electrical actuation force on a droplet}

Jones et al. [40] studied the origins of the electrical force on liquids and modeled the forces using electromechanical frameworks. Models utilizing the Maxwell stress tensor method and simplified RC circuit models were developed to capture the electromechanics involved in the capillary rise of aqueous liquids [42] and the electric field-induced pressure change in an aqueous droplet between parallel plates [41]. Electrowetting and dielectrophoresis were identified as the low- and high-frequency limit response of fluid elements to an external electrical AC signal. Kumari et al. [12] developed similar RC circuit-based electromechanical models to estimate the electrical actuation force on droplets moving between two flat plates. Other researchers [26, 27, 30, 42,43] have developed analytical and numerical capacitance-based models to estimate the actuation force in electrowetting systems. Kuo et al. [23] modeled EW actuation by estimating the EW-induced pressure gradient in terms of the contact angle modification resulting from the application of an EW voltage.

This section uses the electromechanical framework developed by Kumari et al. [12] to highlight the physics underlying each of the three actuation regimes; similar considerations have been employed by Jones [44] and Chatterjee et al. [45] to study frequency-dependent electrical actuation. The framework by Kumari et al. [12] is used to develop a scaling analysis-based model to compare the actuation force in each of the three regimes. For a general case of actuation, the electric field lines penetrate the dielectric layer as well as the droplet. The droplet is sandwiched between the two plates with a separation of $H$ as shown in Figure 2. The top plate and the left electrode on the bottom plate are grounded and the right electrode serves as the actuation electrode. The dielectric thickness covering the bottom electrodes is $h$. Within this framework, the simplest model for studying the actuation of a generic liquid droplet includes the resistance and capacitance of the droplet and those of the dielectric layer. Figure 3 shows a simplified RC model used for the analysis of droplet motion shown in Figure 2. The RC network consists of the dielectric layer and droplet capacitances $C_{d}$ and $C_{D}$, respectively, and the dielectric layer and droplet resistances $R_{d}$ and $R_{D}$, respectively.

The capacitances and resistances in the above circuit can be estimated as:

$$
C_{d}=\frac{A k_{d} \varepsilon_{0}}{h} \text { and } C_{D}=\frac{A k_{D} \varepsilon_{0}}{H}
$$

and

$$
R_{d}=\frac{h}{\sigma_{d} A} \text { and } R_{D}=\frac{H}{\sigma_{D} A}
$$




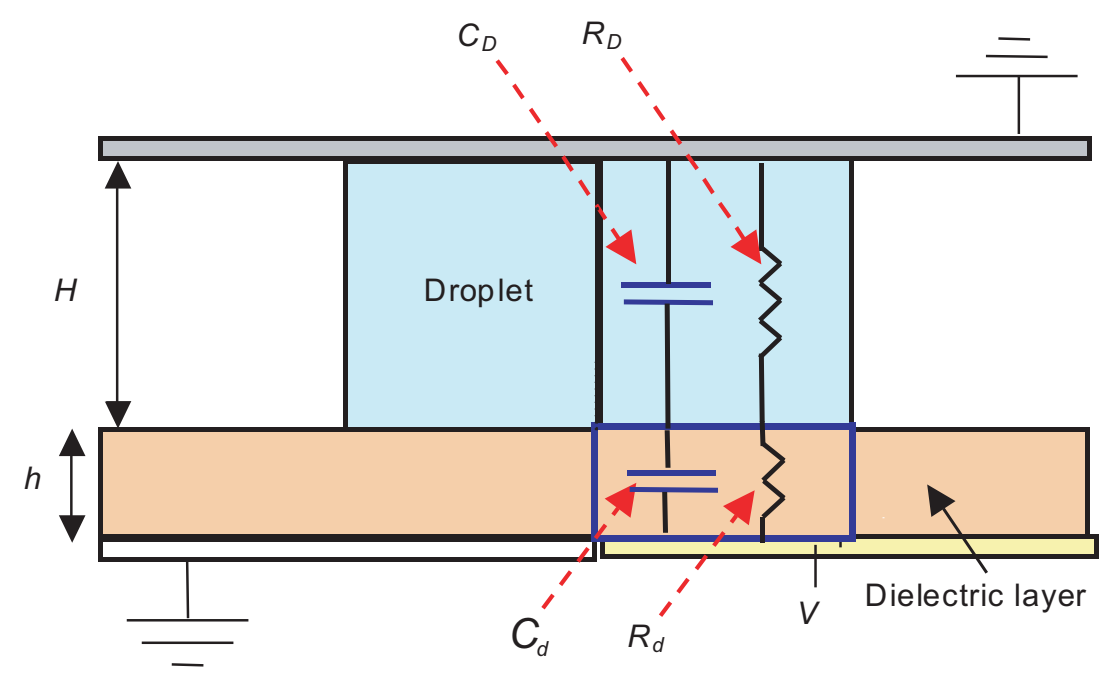

Figure 2. Schematic illustration of a droplet undergoing transition to the actuated electrode on the right [12].

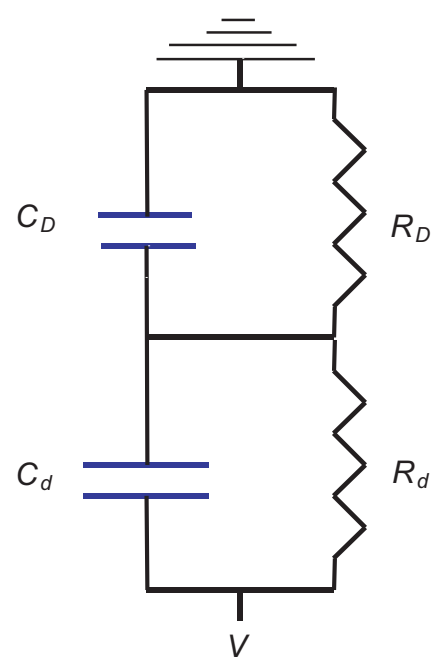

Figure 3. RC network for the analysis of the electrical actuation force [12].

where $\left(k_{d}, \sigma_{d}\right)$ and $\left(k_{D}, \sigma_{D}\right)$ are the (dielectric constant, electrical conductivity) of the dielectric layer and the droplet, respectively, and $A$ is the area of the droplet on the actuated electrode. The total impedances $Z_{d}$ and $Z_{D}$ of the dielectric layer and droplet can be expressed in terms of the frequency $\omega$ of the AC voltage as:

$$
Z_{d}=\frac{R_{d}}{1+j\left(\omega R_{d} C_{d}\right)} \text { and } Z_{D}=\frac{R_{D}}{1+j\left(\omega R_{D} C_{D}\right)}
$$

where $\omega=2 \pi f$ is the angular frequency corresponding to the frequency $f$ of the AC voltage.

The total impedance (sum of the droplet and the dielectric layer impedances) is:

$$
Z_{e q}=Z_{d}+Z_{D}
$$


The total circuit impedance can be used to estimate the effective complex capacitance of the circuit as:

$$
C_{e q}^{*}=\frac{1}{j \omega Z_{e q}}
$$

The above expression is used to estimate the effective capacitance of the system as:

$$
C_{e q}=\frac{\left(\frac{R_{d}^{2} C_{d}}{1+\left(\omega R_{d} C_{d}\right)^{2}}+\frac{R_{D}^{2} C_{D}}{1+\left(\omega R_{D} C_{D}\right)^{2}}\right)}{\left(\left(\frac{R_{d}}{1+\left(\omega R_{d} C_{d}\right)^{2}}+\frac{R_{D}}{1+\left(\omega R_{D} C_{D}\right)^{2}}\right)^{2}+\omega^{2}\left(\frac{R_{d}^{2} C_{d}}{1+\left(\omega R_{d} C_{d}\right)^{2}}+\frac{R_{D}^{2} C_{D}}{1+\left(\omega R_{D} C_{D}\right)^{2}}\right)^{2}\right)}
$$

The total energy stored in the capacitive system is then estimated as:

$$
E=\frac{1}{2} C_{e q} V^{2}
$$

The first derivative of this energy variation yields the actuation force on the droplet as:

$$
F=\frac{d E}{d x}=\frac{1}{2} V^{2} \frac{d C_{e q}}{d x}
$$

Equation (7) represents the frequency-dependent effective system capacitance for the most general case of electromechanical actuation. Kumari et al. [12] showed that Equation (7) reduces to the various regimes of electromechanical actuation depending on the properties of the dielectric layer and the droplet, and the AC frequency.

Case 1) Actuation of dielectric liquids (Insulating droplet and dielectric layer):

This case is defined by $R_{d}=\infty$ and $R_{D}=\infty$. Equation (7) therefore reduces to the following:

$$
C_{e q}=\frac{C_{d} C_{D}}{C_{d}+C_{D}}
$$

The above expression for the equivalent system capacitance corresponds to two capacitors in series. Interestingly, the effective capacitance of the system is independent of the $\mathrm{AC}$ frequency. The actuation force will consequently be independent of the frequency of the AC voltage.

Case 2) AC electrowetting (Electrically conducting droplet and insulating dielectric layer; use of AC voltages):

The mathematical condition for this case is $R_{d}=\infty$, for which Equation (7) reduces to the following:

$$
C_{e q}=\frac{C_{d}\left(1+\omega^{2} C_{d} C_{D} R_{D}^{2}+\left(\omega R_{D} C_{D}\right)^{2}\right)\left(1+\left(\omega R_{D} C_{D}\right)^{2}\right)}{\left(\left(1+\omega^{2} C_{d} C_{D} R_{D}^{2}+\left(\omega R_{D} C_{D}\right)^{2}\right)^{2}+\omega^{2} C_{d}^{2} R_{D}^{2}\right)}
$$

Equation (11) estimates the AC frequency-dependent equivalent system capacitance which can be 
used to calculate the actuation force on droplets during AC electrowetting.

Case 3) Classical EW (Electrically conducting droplet and insulating dielectric layer; use of DC voltages):

DC actuation corresponds to a frequency of zero; under this limit, Equation (7) reduces to:

$$
C_{e q}=C_{d}
$$

The equivalent system capacitance is simply the capacitance of the dielectric layer. A perfectly conducting droplet implies the absence of an electric field inside the droplet; consequently, the entire voltage drop occurs across the dielectric layer.

It should be noted that while Equation (12) for classical EW represents an exact and accurate capacitance of the system, Equations (10) and (11) (for the actuation of dielectric liquids and AC electrowetting, respectively) represent a simplified analysis (which does not account for electric field fringing) to estimate the system capacitance and the actuation force. More detailed modeling can predict the actuation force variation along the length of the transition; such detailed modeling was carried out by Kumari et al. [38] to predict the electrical actuation force on a dielectric droplet. Furthermore, Kumari et al. [12] showed that the actuation force on a droplet (for a specified geometry and actuation voltage) is maximized in the classical EW regime because the entire voltage drop occurs across the dielectric layer. Thus, it is advantageous to use classical EW (to minimize actuation voltages) to actuate a droplet (provided that the droplet electrical conductivity is sufficient to ensure that the entire voltage drop occurs across the dielectric layer).

The three actuation regimes can be more clearly illustrated by comparing the actuation force dependence on the AC frequency for multiple liquids. Kumari et al. [12] quantified the influence of the $\mathrm{AC}$ frequency on the normalized actuation force for three liquids; this normalization was carried out using the classical EW actuation force (which is the maximum possible force). Three aqueous potassium chloride $(\mathrm{KCl})$ solutions of concentrations $2.2 \times 10^{-5} \mathrm{M}, 10^{-4} \mathrm{M}$ and $10^{-3} \mathrm{M}$ were used, with corresponding electrical conductivities of $3.56 \times 10^{-4}, 1.47 \times 10^{-3}$ and $1.47 \times 10^{-2} \mathrm{~S} / \mathrm{m}$, respectively. The actuation force variation (normalized Equation 11) with AC frequency is shown in Figure 4 . For each

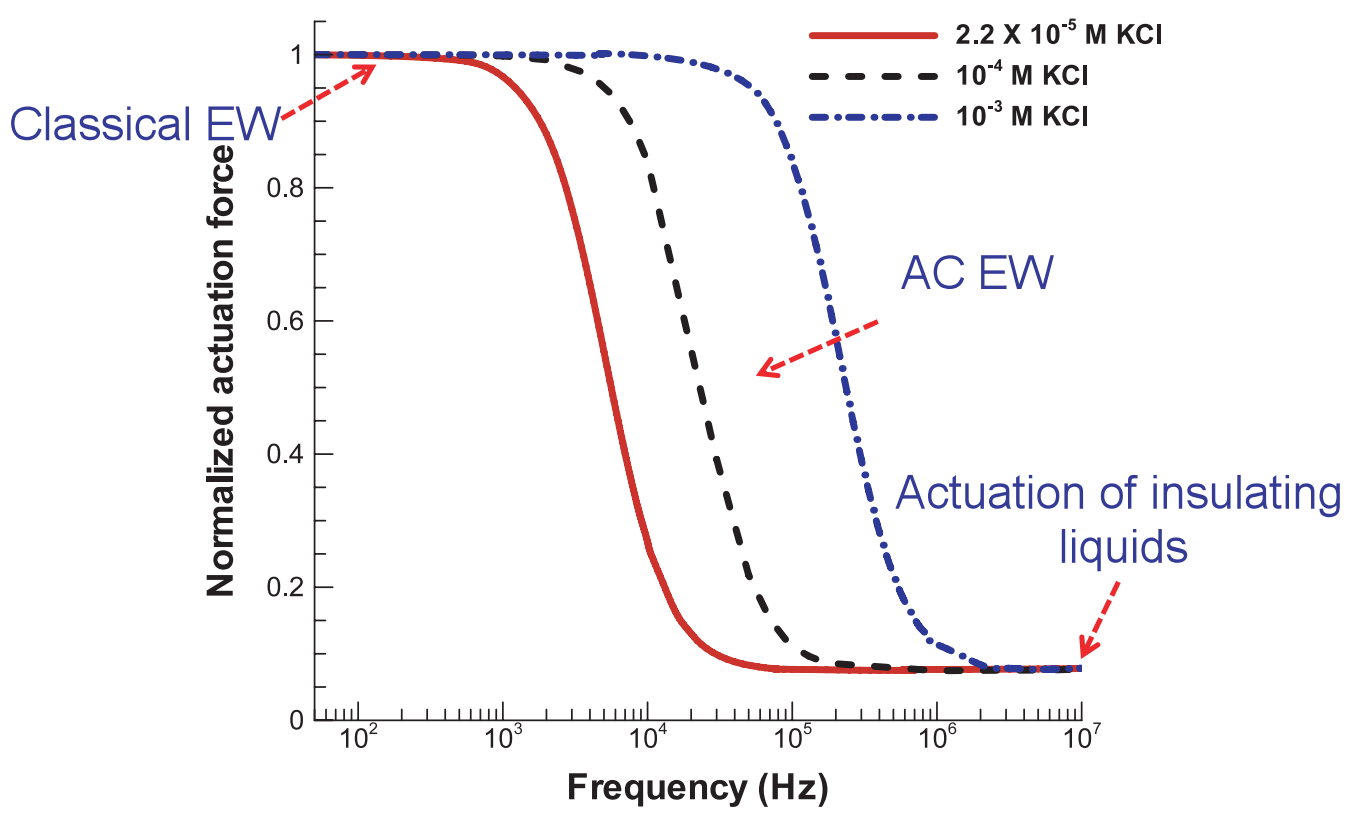

Figure 4. Normalized frequency-dependent actuation force for $\mathrm{KCl}$ solutions [12]. 
liquid, the normalized actuation force is close to 1 at low $\mathrm{AC}$ frequencies; this implies that at low $\mathrm{AC}$ frequencies, the entire voltage drop occurs across the dielectric layer and the actuation force is the same as that for classical EW. As the frequency increases, the normalized actuation force deviates from the classical EW limit when the electric field starts penetrating the droplet. The actuation force then steadily decreases with AC frequency. At sufficiently high AC frequencies, the contribution of the droplet electrical conductivity becomes negligible, and the droplet behaves as electrically insulating; the actuation force does not decrease any further with frequency. This corresponds to the limit of electrical actuation of dielectric liquids. Figure 4 also shows that the electrical conductivity of the droplet strongly determines the frequency at which the force deviates from the EW limit as well as the frequency at which it approaches the limit corresponding to the actuation of dielectric liquids; an increase in the electrical conductivity increases the $\mathrm{AC}$ frequencies required to attain these two limits. It should be noted that similar results can also be obtained using the Maxwell stress tensor method as demonstrated by Jones et al. [42].

\subsection{Modeling droplet motion}

Modeling electrically induced droplet motion presents several challenges since the nature and strength of the forces opposing droplet motion are not clearly understood. Walker and Shapiro [46] developed detailed numerical models to study the fluid dynamics of droplet flows with a particular emphasis on studying the influence of contact angle saturation and contact angle hysteresis (which determines the threshold voltage for EW actuation). Mohseni and Dolatabadi [22] used a Volume-of-Fluid based model to predict transient and steady-state droplet motion, with EW effects introduced in terms of modified contact angles per Lippman's equation [4]. In general, modeling of boundary phenomena [47] comprising contact line friction, contact angle hysteresis and droplet pinning presents several challenges. Several researchers [20, 26, 27, 48, 49] have modeled these effects using scaling arguments or semi-empirical models which rely on experimental data. Modeling the wall stresses resulting from the fluid flow inside a droplet is challenging since the transient velocity profile inside a discrete moving droplet is not clearly understood. Lu et al. [50] recently utilized particle image velocimetry to study the internal fluid dynamics of a droplet under EW-actuation in an air medium. Oprins et al. [51] numerically estimated the velocity profile of a droplet moving under EW-actuation between two flat plates. The presence of a surrounding filler fluid leads to an additional viscous drag on the droplet (typically EW actuation is carried out in air or in an oil environment). This viscous drag has also been modeled semi-empirically [26, 44, 52]. While the presence of an oil carrier medium increases the viscous drag, the contact line friction and the threshold voltage for EW-actuation is reduced in the presence of an oil environment.

Owing to the complexities of modeling the different phenomena influencing droplet motion, simplified analyses have been conveniently employed to model droplet motion [23, 26, 27, 44, 50, 54]. As an illustration, Bahadur and Garimella [26] modeled EW-induced transient droplet motion by representing the opposing forces using simplified algebraic expressions and incorporated them in the overall force balance model for a droplet which led to:

$$
m \frac{d^{2} x}{d t^{2}}=F_{a c t}-F_{w}-F_{f}-F_{c l}
$$

where $m$ is the droplet mass, $F_{a c t}$ is the electrical actuation force, $F_{w}$ is the force resulting from wall stress, $F_{f}$ is the filler fluid drag, and $F_{c l}$ is the contact line friction. Simplified analyses such as those represented by Equation (13) can be used for a first-order estimate of the transient position and velocity of a droplet as it moves under electrical actuation. 
Table 1. Summary of experimental results on electrically induced droplet motion.

\begin{tabular}{|c|c|c|c|c|}
\hline Study & Fluid & Voltage & $\begin{array}{l}\text { Dielectric layer } \\
\text { specification }\end{array}$ & $\begin{array}{c}\text { Measured } \\
\text { droplet } \\
\text { velocity }(\mathrm{cm} / \mathrm{s}) \\
\end{array}$ \\
\hline \multicolumn{5}{|c|}{ Classical electrowetting } \\
\hline $\begin{array}{l}\text { Kumari et al. } \\
\text { [12], } 2008\end{array}$ & $\begin{array}{c}\mathrm{KCl} \text { solutions }(2.2 \times \\
10^{-5} \mathrm{M}, 10^{-4} M \text { and } \\
\left.10^{-3} M\right)\end{array}$ & $50 V \mathrm{DC}$ & $\begin{array}{c}0.8 \mu \mathrm{m} \\
\text { Parylene C }\end{array}$ & 4.2 \\
\hline $\begin{array}{l}\text { Pollack et al. } \\
\text { [20], } 2002\end{array}$ & $\begin{array}{c}\mathrm{KCl} \text { solutions }(1- \\
\left.10^{-3} \mathrm{M}\right)\end{array}$ & $\begin{array}{c}15-100 V \\
\text { DC }\end{array}$ & $\begin{array}{c}0.8 \mu \mathrm{m} \\
\text { Parylene C }\end{array}$ & 10 \\
\hline $\begin{array}{l}\text { Kuo et al. [23], } \\
\qquad 2003\end{array}$ & $\begin{array}{c}\text { DI water, } \mathrm{KCl} \\
\text { solution }(0.1 \mathrm{M})\end{array}$ & $600 V \mathrm{DC}$ & $38 \mu m$ PDMS & 0.4 \\
\hline $\begin{array}{l}\text { Lee et al. [28], } \\
2002\end{array}$ & Water & $15 V \mathrm{DC}$ & $\begin{array}{c}0.07 \mu m \text { Barium } \\
\text { Strontium } \\
\text { Titanate }\end{array}$ & Not reported \\
\hline \multicolumn{5}{|c|}{ AC electrowetting } \\
\hline $\begin{array}{l}\text { Kumari et al. } \\
\text { [12], } 2008\end{array}$ & $\begin{array}{c}\mathrm{KCl} \text { solutions }(2.2 \times \\
10^{-5} M, 10^{-4} M \text { and } \\
\left.10^{-3} M\right)\end{array}$ & $\begin{array}{l}50 \mathrm{V@0} \text { - } \\
450 \mathrm{kHz}\end{array}$ & $\begin{array}{c}0.8 \mu m \\
\text { Parylene C }\end{array}$ & $\begin{array}{c}0-7.4 \\
\text { (frequency } \\
\text { dependent) }\end{array}$ \\
\hline $\begin{array}{c}\text { Baviere et al. } \\
\text { [35], } 2008\end{array}$ & $\begin{array}{l}\text { Water-glycerol } \\
\text { mixtures }\end{array}$ & $\begin{array}{l}95 V @ \\
600 H z\end{array}$ & $1.3 \mu \mathrm{m} \mathrm{Si}_{3} \mathrm{~N}_{4}$ & 16 \\
\hline $\begin{array}{l}\text { Chatterjee et } \\
\text { al. [29], } 2006\end{array}$ & $\begin{array}{l}29 \text { liquids } \\
\text { comprising } \\
\text { aqueous solutions, } \\
\text { organic solvents } \\
\text { and ionic liquids }\end{array}$ & $\begin{array}{c}90 \mathrm{V@8} 8 \\
k H z\end{array}$ & $\begin{array}{c}0.98 \mu m \\
\text { Parylene } \mathrm{C} \text { or } \\
1.4 \mu m \mathrm{SiO}_{2}\end{array}$ & Not reported \\
\hline $\begin{array}{c}\text { Cooney et al. } \\
{[30], 2006}\end{array}$ & $\begin{array}{c}\mathrm{KCl} \text { solutions }(0-1 \\
M)\end{array}$ & $\begin{array}{l}73 \mathrm{V@} \\
100 \mathrm{~Hz}\end{array}$ & $\begin{array}{c}1 \mu m \text { Parylene } \\
\text { C }\end{array}$ & 3 \\
\hline $\begin{array}{l}\text { Yi and Kim } \\
{[31], 2006}\end{array}$ & DI water & $\begin{array}{c}65 V @ 1 \\
\mathrm{kHz}\end{array}$ & $0.3 \mu m \mathrm{SiO}_{2}$ & 1 \\
\hline $\begin{array}{l}\text { Torkkeli et al. } \\
\text { [32], } 2001\end{array}$ & DI water & $\begin{array}{c}124 V @ 1 \\
\mathrm{kHz}\end{array}$ & $\begin{array}{c}65 \mu \mathrm{m} \\
\text { cellulose } \\
\text { acetate tape }\end{array}$ & 1 \\
\hline $\begin{array}{c}\text { Washizu [36], } \\
1998\end{array}$ & DI water & $\begin{array}{c}400 \mathrm{V@} \\
60 \mathrm{~Hz}\end{array}$ & $\begin{array}{c}10 \mu \mathrm{m} \\
\text { photoresist }\end{array}$ & Not reported \\
\hline \multicolumn{5}{|c|}{ Actuation of dielectric liquids } \\
\hline $\begin{array}{c}\text { Kumari et al. } \\
\text { [38], } 2008\end{array}$ & Transformer oil & $1000 \mathrm{~V}$ & $\begin{array}{c}3 \mu m \text { Parylene } \\
\mathrm{C}\end{array}$ & 0.08 \\
\hline $\begin{array}{l}\text { Kuo et al. [23], } \\
2003\end{array}$ & Olive oil & $600 \mathrm{~V}$ & $38 \mu m$ PDMS & 0.03 \\
\hline
\end{tabular}

\subsection{Characteristics of droplet motion}

Table 1 presents a summary of results from available experiments on electrical actuation of droplets between flat plates or on a single plate. It should be noted that several other researchers have studied different aspects of electrical actuation on smooth surfaces. However the focus of the present work is the analysis of the actuation forces and velocities during droplet motion; this is the basis for selection of the entries in Table 1. Table 1 arranges the available results according to the three electromechanical regimes discussed above and shows the fluid, the actuation voltage, the dielectric layer details and the experimentally measured droplet velocities. This section summarizes key observations and results associated with electrical actuation of droplets in each of the three electromechanical regimes. 


\subsubsection{Classical electrowetting}

Classical electrowetting has been the most studied form of electrical actuation; key features associated with classical EW-induced droplet motion are as follows:

1. Classical EW is based on the premise of the droplet being a perfect electrical conductor and the use of DC voltages for actuation; classical EW can also be interpreted as the low-frequency response of a fluid element to an electrical signal as discussed by Jones et al. [42].

2. Classical EW is an electromechanical phenomena and is not a consequence of solid-liquid interfacial energy decrease or the associated contact angle change as implied by Lippman's [4] equation.

3. The actuation force in classical EW is greatest among the three regimes (for a specified actuation voltage and geometry). In other words, the actuation voltage for a specified liquid is the lowest if the droplet is moved in the classical EW regime.

4. The actuation force on the droplet depends only on the underlying dielectric layer properties as the entire voltage drop occurs across the dielectric layer. The actuation force is directly proportional to the dielectric constant and inversely proportional to the thickness of the dielectric layer. The lower limit of dielectric layer thickness is, however, influenced by other considerations such as the occurrence of pinholes, dielectric breakdown and chemical resistance.

5. The practice of adding salt to water to make it electrically conducting is not needed from an actuation force perspective. Pure water picks up ions from the atmosphere and the resulting conductivity ensures that the entire voltage drop still occurs across the dielectric layer. The lack of influence of salt concentration on droplet actuation has been experimentally reported by several researchers $[20,23,26,30,32]$.

6. Higher droplet velocities have been obtained upon elimination of the top plate and grounding of the droplet from the side by multiple researchers [30,31]; this can be attributed to the elimination of the opposing forces associated with the top plate. It is important to note that the droplet still must be grounded to ensure a complete transition to the actuated electrode.

7. Table 1 shows that droplet velocities of up to $10 \mathrm{~cm} / \mathrm{s}$ have been obtained using aqueous solutions. The droplet velocity shows a strong parabolic dependence on the actuation voltage. An upper limit on the actuation voltage is subject to practical considerations such as dielectric breakdown and droplet instability. It should also be noted that a weak dependence of the droplet velocity on the droplet size (for the same actuation voltage) has been reported [20,26]; this implies that the use of smaller droplets will not necessarily increase the rate of microfluidic operations.

8. The use of oil as a filler fluid reduces the threshold voltage required for actuation considerably [54] and also influences the boundary phenomena at the triple phase line which determine droplet velocity.

\subsubsection{AC electrowetting}

AC electrowetting has been well-investigated and is associated with several interesting phenomena; key features associated with AC EW-induced droplet motion are as follows:

1. The actuation force in this regime depends on the electrical properties of the droplet and of the dielectric layer, as well as the AC frequency. In this regime, there is a finite electric field inside the droplet as well as in the dielectric layer.

2. The regime of AC EW reduces to the classical EW regime at low frequencies and the dielectric liquid actuation regime at high frequencies.

3. AC EW is widely used in the available studies on droplet actuation despite the lower forces that results compared to classical EW. Key advantages include the reduced possibility of chemical reactions [29], and lower contact angle hysteresis upon the use of AC voltages [37].

4. Experimentally reported velocities are of the same order of magnitude as those obtained with classical EW-induced motion. Kumari et al. [12] conducted a direct comparison of droplet 
velocities of aqueous $\mathrm{KCl}$ solutions under $\mathrm{AC} \mathrm{EW}$ and classical EW using the same experimental setup. The experimentally measured velocities under low AC frequency actuation were up to $70 \%$ higher than the velocities under classical EW actuation using the same voltage. The actuation force under low-frequency AC EW would be the same as that under classical EW actuation; these results therefore imply that the use of $\mathrm{AC}$ voltages reduces the forces opposing droplet motion. This can be attributed to lower contact line friction under AC EW, as also reported by Berge and Peseux [37], in the form of reduced contact angle hysteresis upon the use of AC voltages. However, the influence of AC voltages on the opposing forces is not completely understood and requires further investigation.

5. The available experimental results and analyses suggest that low-frequency AC electrowettingbased actuation can offer the combined benefits of a high actuation force, reduced opposing forces and increased device reliability, all of which are highly desirable features for the design of practical microfluidic systems.

\subsubsection{Actuation of dielectric liquids}

Actuation of dielectric droplets has received very little attention in the available literature; key features associated with the motion of dielectric liquid droplets are as follows:

1. The actuation force on a dielectric droplet can be estimated by calculating the capacitance of the liquid and the underlying dielectric layer in series (the dielectric layer is not a strict requirement for the actuation of dielectric liquids).

2. The actuation force on a dielectric liquid does not depend on the frequency of the AC voltage.

3. Electrically conducting liquids can also be actuated in this regime by using an AC voltage of sufficiently high frequency (depending on the electrical conductivity of the droplet); thus this regime is the high-frequency response of the liquid to an electrical signal.

4. Experimental results indicate that dielectric liquid actuation requires voltages on the order of $1000 \mathrm{~V}$. This is a consequence of the voltage drop occurring across the entire distance between the parallel plates (i.e., across the height of the droplet).

5. Experimentally available droplet velocities are two orders of magnitude lower than in the other two regimes discussed above. It should be noted that dielectric liquids are typically low surface tension liquids and have higher wettability than electrically conducting liquids. Thus a fluoropolymer layer which leads to hydrophobicity using an aqueous liquid will lead to oleophilicity for a dielectric liquid; this will increase the friction force on the dielectric droplet considerably.

\subsection{Avenues for future research}

Significant progress has been made in understanding the fundamental physics underlying electrical actuation of droplets. The performance of electrical actuation systems is influenced by several secondary phenomena which include contact angle saturation, role of the electrical double layer, satellite droplet emission and charge trapping inside the dielectric layer; current understanding of these phenomena is limited. Further challenges to the study of droplet-based systems arise from phenomena occurring at the triple phase line; contact line friction and droplet pinning are incompletely understood and pose significant challenges to modeling. The transient velocity profile in a discrete moving droplet also needs to be well understood to estimate the viscous forces acting on the droplet. The presence of oil as a filler fluid in such systems affects the droplet dynamics favorably by reducing friction and the threshold voltage for actuation; the role of an oil medium in enhancing droplet transport rates needs to be studied in greater detail. Progress in the fabrication of ultra-high dielectric constant thin films [53] offers opportunities to reduce the actuation voltages by an order of magnitude and bring them into the $1 \mathrm{~V}$ range.

A majority of the available work on the electrical actuation of liquid droplets has considered electrically conducting liquid droplets; a few recent efforts have demonstrated the feasibility of using 
electrical techniques to actuate insulating liquids. There is tremendous potential for extending the range of liquids and developing compatible microfluidic actuation techniques for insulating liquids, solvents, biological fluids, colloidal suspensions and ionic liquids using electrical and other actuation force fields (chemical, optical, thermal, magnetic). This will increase the types of liquids in the microfluidics toolkit which are amenable to control and could result in the development of several novel lab-on-achip and micro-reactor applications.

\section{ELECTROWETTING-BASED CONTROL OF DROPLET STATES AND TRANSITIONS ON SUPERHYDROPHOBIC SURFACES}

\subsection{Droplet states and transitions on structured surfaces}

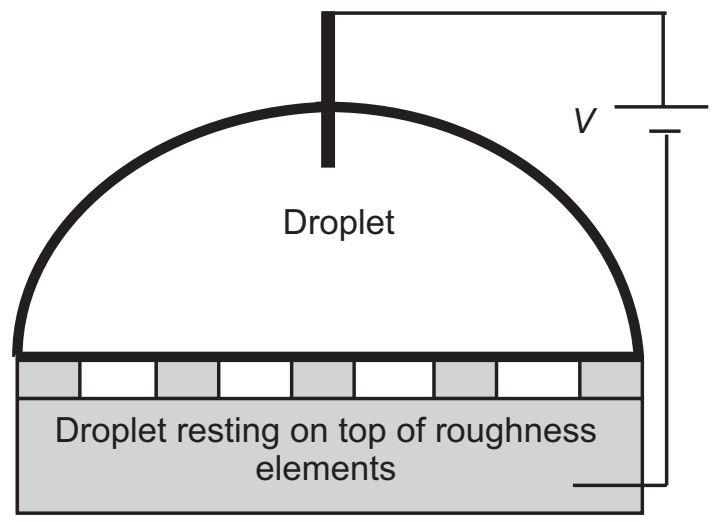

(a)

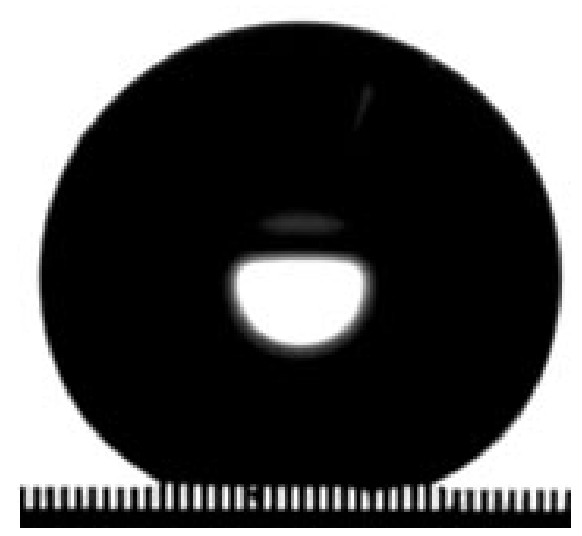

(c)

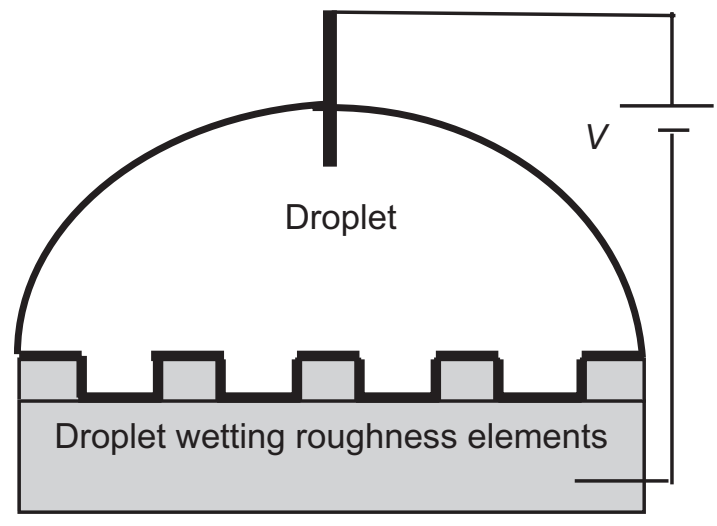

(b)

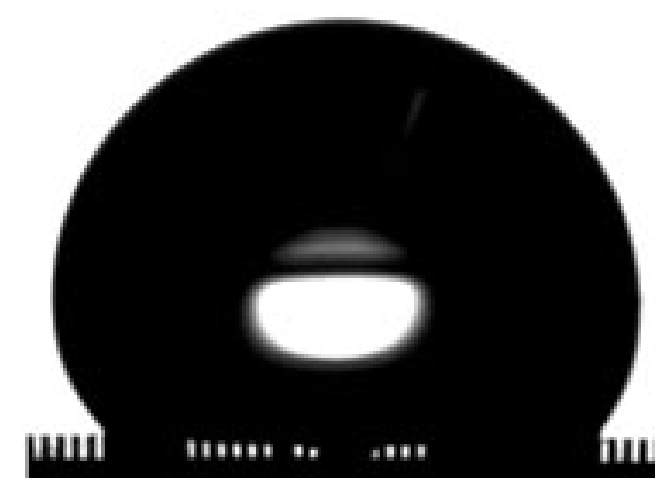

(d)

Figure 5. Droplet states on structured surfaces [56]: schematic illustration of (a) Cassie state, and (b) Wenzel state. (c) A $2 \mathrm{~mm}$ diameter water droplet in the Cassie state (light passing between pillars below droplet) in the absence of an EW voltage, and (d) droplet in the Wenzel state at an EW voltage of $80 \mathrm{~V}$.

The study of the interaction of a static droplet with structured surfaces generally involves the analysis of the droplet in two extreme configurations on the surface. In the Cassie state [55] (Figure 5a) the droplet base rests only on the tips of the roughness elements; consequently the droplet is in composite contact with air and solid at its base. In the Wenzel state [56] (Figure 5b) the droplet fills the space between the roughness elements and is in intimate contact with the surface. The classical Cassie and Wenzel equations highlight the role of surface structuring in altering the macroscopic (apparent) 
contact angle of the droplet and changing the effective solid-liquid contact area. Since EW is also a powerful tool for influencing the contact angles, appropriate surface structuring can be used in conjunction with EW voltages for enhanced control of droplet states and transitions. Figures 5 (c-d) show direct visual confirmation of the EW-induced Cassie-Wenzel transition on microstructured superhydrophobic pillared surfaces. Figure 5c shows the Cassie state (in the absence of an EW voltage), as is evident from the light passing between the pillars. Application of a sufficient EW voltage switches the droplet to the Wenzel state shown in Figure 5d. It should be noted that EW on structured surfaces requires the roughness elements to be coated with a conformal thin dielectric layer. The dielectric layer is then coated with a thin conformal fluoropolymer layer to impart superhydrophobicity to the surface.

Droplet states and transitions have been studied by many researchers with the objective of developing nonwetting and low-adhesion surfaces which mimic the self-cleaning properties associated with the lotus leaf structure [57-59]. Researchers have carried out extensive experiments and modeling to study droplet states and transitions on artificially structured superhydrophobic surfaces. Patankar [60] showed that the Cassie and Wenzel droplets differ in their surface energies, and that the stable equilibrium state of the droplet corresponds to the state which has a lower energy. He et al. [61] demonstrated Cassie and Wenzel droplet formation on the same surface depending on the way the droplets are formed. Patankar [62] also analyzed the Cassie-Wenzel transition of a droplet and discussed the presence and magnitude of an energy barrier for the Cassie-Wenzel transition. In addition to energy-based analyses, the Cassie-Wenzel transition has also been studied using geometry-based models [63-65] which rely upon the use of the Laplace equation and the calculation of meniscus curvatures to predict transition. However, energy minimizationbased analyses are more appropriate for analyzing droplet transition as they rely on the estimation of interfacial areas which are much easier to compute than meniscus curvatures. The present work focuses on the use of energy minimization-based analyses for the study of EW-induced droplet state control.

The influence of an EW voltage on droplet behavior on microstructured [18, 56, 66-68] and nanostructured [69-76] surfaces has received significant research interest in the last five years. Krupenkin et al. [71] and Ahuja et al. [78] demonstrated EW-induced Cassie-Wenzel transition of droplets of aqueous $\mathrm{KCl}$ and organic solvents on superhydrophobic nanostructured surfaces. Bahadur and Garimella [18] developed analytical models and conducted experiments [56,70] to understand different aspects of the Cassie-Wenzel transition and to analyze the reasons for the lack of reversibility of the transition. The present work summarizes theoretical and experimental results from existing studies of EW-induced control of droplet states and transitions on superhydrophobic surfaces.

\subsection{Understanding the Cassie-Wenzel transition}

The contact angle of a droplet in the Cassie state depends on a dimensionless surface parameter $\phi$ which is defined as the ratio of the area of the top of the pillars to the total base area of the substrate. The apparent contact angle $\theta_{C}$ of a droplet in the Cassie state can be obtained [18] from the energyminimization principle as:

$$
\cos \theta_{C}=-1+\phi\left(1+\cos \theta_{0}\right)
$$

where $\theta_{0}$ is the contact angle of the droplet on a flat surface. The hydrophobicity of a Cassie droplet increases as $\phi$ decreases because of enhanced contact with air. The contact angle of a droplet in the Wenzel state depends on the surface roughness $r_{m}$ (dimensionless) which is defined as the ratio of the total surface area (including the sides and base) of the roughness elements to the projected surface area (not including the sides) of the roughness elements. For a Wenzel droplet, the apparent contact angle $\theta_{W}$ is obtained [18] as:

$$
\cos \theta_{W}=r_{m} \cos \theta_{0}
$$

The above equation shows that the Wenzel state amplifies the initial hydrophobicity or hydrophilicity of the droplet. 
Bahadur and Garimella [18] developed energy minimization-based models to predict the apparent contact angle of a droplet resting on a structured surface in the presence of an EW voltage. In the analysis, the substrate and the roughness elements are assumed electrically conducting, and the roughness elements have a conformal coating of a thin dielectric layer of thickness $d$ and dielectric constant $k$. An EW field is assumed to be established across the dielectric by means of electrodes contacting the electrically conducting droplet and the substrate. The model consists of minimizing the total surface energy content of the droplet, which comprises the solid-liquid, liquid-air and solid-air interfacial energies and the associated areas; the effect of the EW field is incorporated using Lippman's equation [4] which estimates the reduction in the solid-liquid interfacial energy upon the application of an EW voltage. The apparent contact angle $\theta_{C}^{E}$ of a droplet in the Cassie state under the influence of an EW voltage $V$ is [18]:

$$
\cos \theta_{C}^{E}=-1+\phi\left(1+\cos \theta_{0}+\eta\right)
$$

where $\eta$ is the electrowetting number expressed as:

$$
\eta=\frac{k \varepsilon_{0} V^{2}}{2 d \gamma_{L A}^{0}}
$$
Sim:

$$
\cos \theta_{W}^{E}=r_{m}\left(\cos \theta_{0}+\eta\right)
$$

Patankar [62] showed that the surface energy of a droplet can be expressed solely in terms of the apparent contact angle. In addition, the droplet surface energy increases with the apparent contact angle of the droplet. This implies that the lower of the Cassie and Wenzel angles corresponds to the stable equilibrium position of the droplet. Since EW affects the energy content of the droplet, it can be used as a tool for controlling the Cassie and Wenzel states. The structured surface needs to be designed such that the Cassie state is more favorable energetically than the Wenzel state in the absence of an EW voltage. Transition to the Wenzel state can be achieved by the application of a suitable EW voltage such that the electrowetted Wenzel state is lower in energy than the electrowetted Cassie state. This is the basic principle underling the dynamic tuning of droplet states on superhydrophobic surfaces.

The energy minimization framework has been further used by Bahadur and Garimella [18] to analyze the Cassie-Wenzel transition in detail by estimating the energy content of the intermediate transition states. The energies of the intermediate transition states relative to the Cassie and Wenzel states determine the presence of an energy barrier to the Cassie-Wenzel transition. The analysis indicates that the EW voltage determines the existence and strength of the energy barrier which needs to be overcome for the Cassie-Wenzel transition. Table 2 summarizes the influence of an EW voltage in controlling droplet states on structured surfaces. For any specified surface $\left(r_{m}\right.$ and $\left.\phi\right)$ and a specified fluid (contact angle $\theta_{0}$ on a flat surface), the magnitude of the EW voltage determines the relative stability of the Cassie and Wenzel states and the energy barrier for transition between the two states. Table 2 shows that the minimum electrowetting number required for a transition to the Wenzel state is:

$$
\eta=-\cos \theta_{0}-\frac{(1-\varphi)}{\left(r_{m}-\varphi\right)}
$$

Also, Table 2 also indicates the existence of a finite energy barrier to the Cassie-Wenzel transition above the minimum electrowetting number estimated by Equation (19). Droplet transition using a voltage obtained per Equation (19) will therefore require additional activation energy for the droplet to successfully overcome this energy barrier. This requirement of activation energy can be eliminated if the EW number $\eta$ is further increased such that: 


$$
\eta=-\cos \theta_{0}
$$

It is also seen that the transition voltage obtained using Equation (20) is independent of surface morphology $\left(r_{m}\right.$ and $\left.\phi\right)$.

Table 2. Influence of the EW number in controlling droplet states on structured surfaces [18].

\begin{tabular}{|c|c|c|}
\hline EW number & Stable state & Comments \\
\hline$\eta<-\cos \theta_{0}-\frac{(1-\phi)}{(r-\phi)}$ & Cassie & $\begin{array}{l}\text { Finite energy barrier for Cassie-Wenzel transition. Barrier } \\
\text { for reverse transition lower. }\end{array}$ \\
\hline$\eta>-\cos \theta_{0}-\frac{(1-\phi)}{(r-\phi)}$ & Wenzel & $\begin{array}{l}\text { Finite energy barrier for Cassie-Wenzel transition. Barrier } \\
\text { for reverse transition higher. }\end{array}$ \\
\hline$\eta>-\cos \theta_{0}$ & Wenzel & $\begin{array}{l}\text { No energy barrier for Cassie-Wenzel transition. Reverse } \\
\text { transition disallowed. }\end{array}$ \\
\hline
\end{tabular}

The above concepts were illustrated by Bahadur and Garimella [18] using a surface with roughness $r_{m}=3$ and the fraction of solid contacting the liquid $\phi=0.5$. The contact angle $\theta_{0}$ of the droplet on a flat surface without an EW voltage is assumed to be $115^{\circ}$ (this corresponds to a water droplet on Teflon). According to equation (19) the electrowetted Wenzel state is lower in energy than the electrowetted Cassie state if $\eta>0.22$. Figure 6a shows the dimensionless droplet surface energy plotted as a function of the effective roughness $r$ for the case when $\eta=0.3$. (The effective roughness $r$ is a measure of the amount of wetting with the condition $1 \leq r \leq r_{m} ; r=1$ corresponds to the electrowetted Cassie state whereas $r=r_{m}$ corresponds to the electrowetted Wenzel state). The contact angle at an intermediate transition state can be estimated by the energy-minimization principle [18] as:

$$
\theta_{t r}^{E}=\cos ^{-1}\left((r-1+\phi)\left(\cos \theta_{0}+\eta\right)-1+\phi\right)
$$

Figure 6a shows that the droplet surface energy increases as the droplet progressively wets the grooves. The maximum surface energy in Figure 6a corresponds to the position where the droplet completely wets the sidewalls of all the grooves and is about to contact the base of the grooves. When the droplet contacts the base of the grooves, the droplet energy is suddenly lowered to the value of the electrowetted Wenzel state. The droplet energy change from $r=1$ to $r=r_{m}$ is continuous, corresponding to a steady change in solid-liquid interfacial area. However at $r=r_{m}$ there is a step change in the solidliquid interfacial area; this leads to a step change in the total surface energy as well. Figure 6b shows the droplet transition configuration plot on the same surface for $\eta=0.5$ which satisfies Equation (20) since $\left(\eta+\cos \theta_{0}\right)>0$. The droplet energy decreases with progressive wetting of the grooves and there is a step jump corresponding to the wetting of the base of the grooves. There is no activation energy required for the Cassie-Wenzel transition as the natural tendency of such a system is to spontaneously transition to the lower-energy Wenzel state.

The droplet transition analysis developed by Bahadur and Garimella [18] is the basis for dynamic control of droplet states by electrowetting. The surface design should ensure that the Cassie state is lower in energy than the Wenzel state in the absence of an EW voltage. The surface roughness parameters can be chosen subject to the following restrictions emerging from equations (14) and (15):

$$
\cos \theta_{0}<-\frac{(1-\phi)}{\left(r_{m}-\phi\right)}
$$




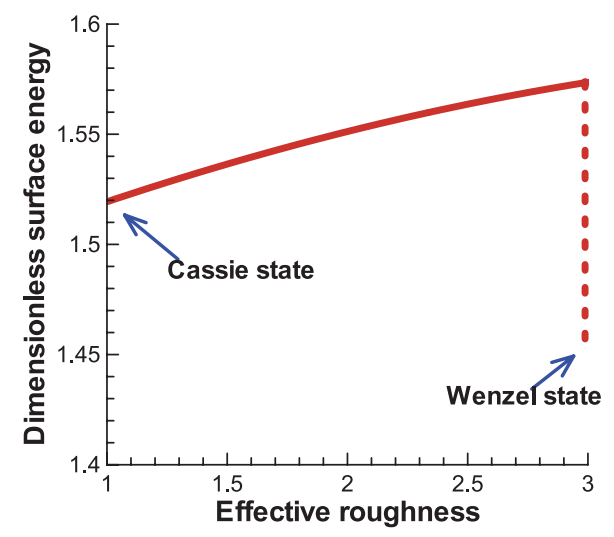

(a)

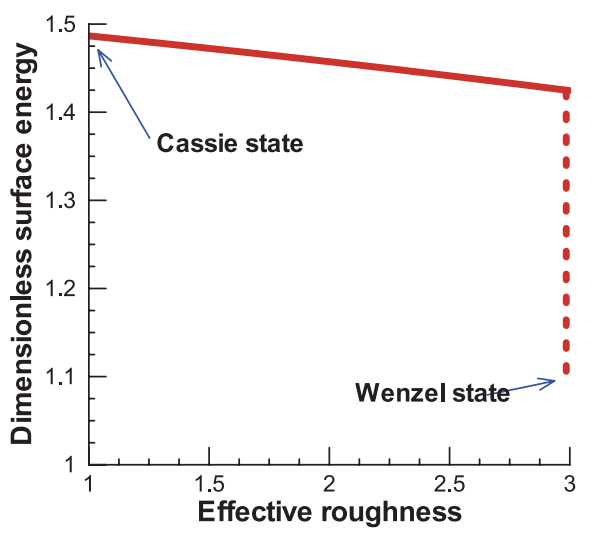

(b)

Figure 6. EW-induced droplet transition energy plot a) with an energy barrier, and (b) without an energy barrier [18].

$$
\text { and } \theta_{0}>90^{\circ} \text {. }
$$

The Wenzel transition can be achieved by applying a minimum EW voltage obtained using Equation (19). However the droplet will still require additional activation energy to transition to the Wenzel state. This requirement of activation energy is eliminated if the EW number $\eta$ is increased such that it satisfies Equation (20).

Various aspects of the modeling presented in this section have been benchmarked against experimental studies of the Cassie-Wenzel transition on micro- and nano-structured surfaces. Krupenkin et al. [71] experimentally studied the EW-induced transition of high surface tension liquids (water and molten salt) on nanostructured silicon surfaces. During the experiments, a sharp CassieWenzel transition was observed at $22 V$ for two different nanostructured surfaces; this transition voltage was seen to be independent of surface morphology $\left(r_{m}\right.$ and $\left.\phi\right)$. Ahuja et al. [78] demonstrated EWinduced Cassie-Wenzel transition of low surface tension organic liquids (n-butanol, cyclopentanol, $\mathrm{n}$ octanol and ethanol) on specially fabricated nanostructured surfaces (referred to as 'nanonails') with overhangs on top of the roughness pillars; a sharp Cassie-Wenzel transition was again seen. Bahadur and Garimella [56] conducted extensive experiments to measure the EW voltage required to trigger the Cassie-Wenzel transition on microstructured surfaces. A sharp Cassie-Wenzel transition was reported at a particular EW voltage for surfaces with the same dielectric layer thickness; this voltage was also independent of surface morphology $\left(r_{m}\right.$ and $\left.\phi\right)$. The reported sharp transition to the Wenzel state [56, $71,78]$ and the independence of the EW voltage on surface parameters is a direct validation of Eq. (20) which predicts EW transition without the requirement of any additional activation energy.

The models presented in this section can also be used to analyze the reversibility of the CassieWenzel transition as described later in this article. In particular, the models can be used to estimate the strength of the energy barrier for the Cassie-Wenzel transition and the reverse transition. However the models have limited utility in predicting droplet contact angles in the electrowetted Wenzel state. Herbertson et al. [68] measured the EW-induced contact angle change of a water droplet in the Wenzel state on pillared surfaces (with pillars of circular cross section); the models developed by Bahadur and Garimella [18] showed a reasonable match with the results of Herbertson et al. [68]. However, the models did not match similar measurements of Bahadur and Garimella [56] of the EW-induced contact angle change in the Wenzel state on pillared surfaces (with pillars of square cross section). The structured surfaces utilized by Herbertson et al. [68] and Bahadur and Garimella [56] had similar surface parameters; the important difference was the shape of the pillars (circular [68] versus square [56]). These results indicate that energy minimization-based models have limited utility in predicting 
droplet behavior in the Wenzel state; this can be attributed to the dominance of nonconservative dissipative forces in the Wenzel state. These forces comprise the contact line pinning force [77,78], contact line friction $[26,54]$ and viscous shear. The strength of the dissipative forces depends on the specific material-fluid combination and the geometry of the structured surface; the difference in the shape of the pillars used by Herbertson et al. [68] and Bahadur and Garimella [56] could explain the difference in the experimental results.

\subsection{Reversibility of the Cassie-Wenzel transition}

A key characteristic in the study of droplet transitions on structured surfaces concerns the reversibility of the EW-induced Cassie-Wenzel transition upon removal of the EW voltage. The most significant observation from available studies on reversibility is the lack of complete reversibility of the CassieWenzel transition in the absence of any active stimulus to revert to the Cassie state.

Bahadur and Garimella [56] identified two key factors inhibiting the natural reversibility of the Cassie-Wenzel transition. The first is the energy barrier [64, 66, 72] to the Wenzel-Cassie transition upon the removal of the EW voltage. Appropriate surface design can ensure that the Cassie state is energetically favored relative to the Wenzel state in the absence of an EW voltage; however, there still exists a barrier for the Wenzel-Cassie transition. This energy barrier is shown in Figure 7 which shows the variation of droplet energy as it progressively wets the grooves. The droplet surface energy is measured in terms of the droplet contact angle (an increase in the contact angle increases the droplet energy). The surface considered in Figure 7 has a roughness $r_{m}$ of 1.91 and $\phi$ of 0.41 . The lower curve in Figure 7 shows the energy content of the droplet as it transitions to the electrowetted Wenzel state at an EW voltage represented by $\eta=0.55$ (corresponding to $60 \mathrm{~V}$ for a water droplet on a surface with a dielectric thickness of $0.8 \mu \mathrm{m}$ ). The downward slope of the lower curve in Figure 7 indicates a spontaneous EW-induced Wenzel transition. The upper curve in Figure 7 shows the energy content variation of the droplet for the reverse transition upon removal of the EW voltage. It is seen that the droplet needs activation energy to overcome the energy barrier for the Wenzel-Cassie transition. This requirement of activation energy is one factor inhibiting the reversibility of the Cassie-Wenzel transition.

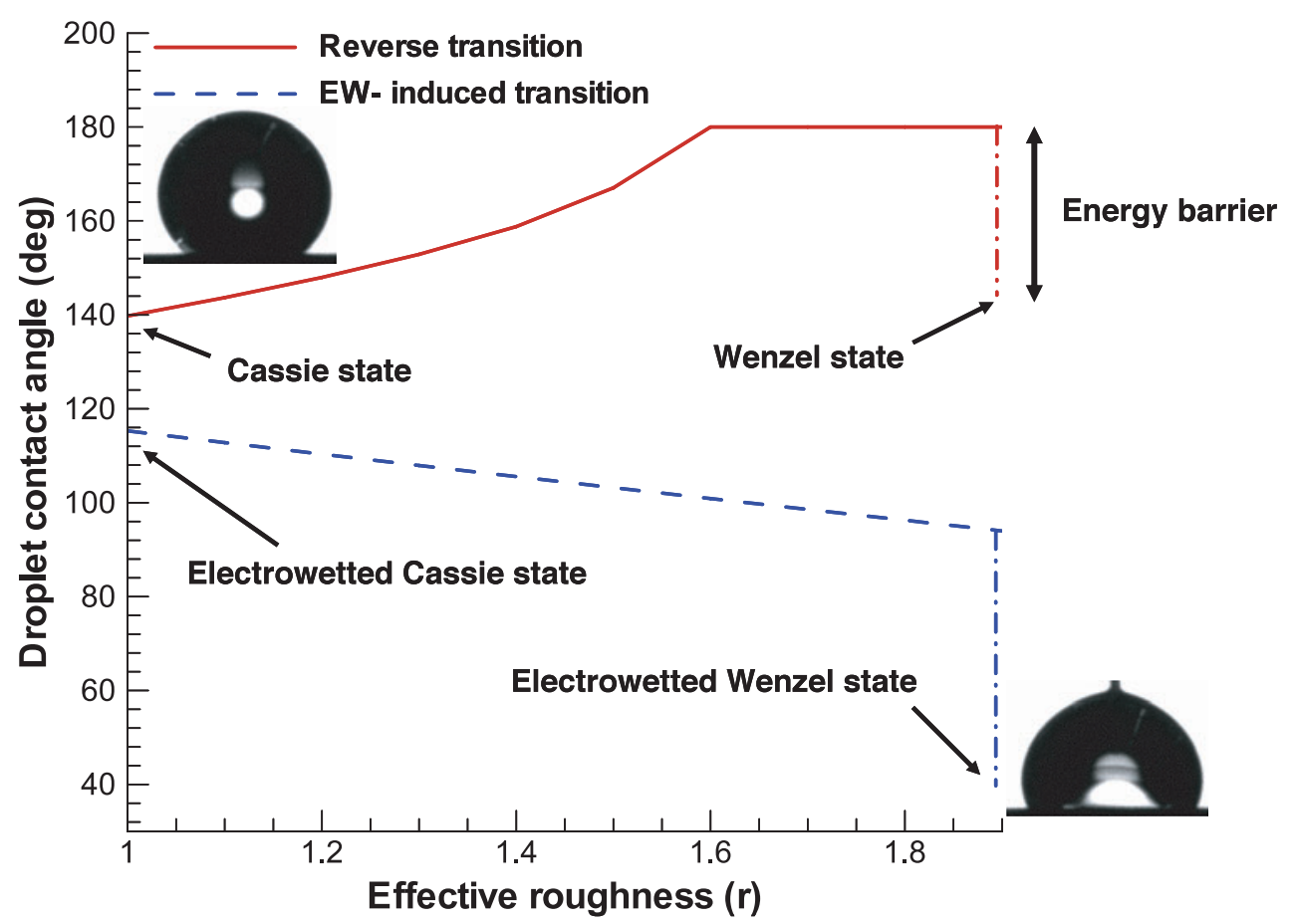

Figure 7. Surface energy content of a water droplet during transition $\left(r_{m}=1.91\right.$ and $\left.\phi=0.41\right)$. The lower curve shows the surface energy variation during the EW-induced Cassie-Wenzel transition, while the upper curve shows the surface energy variation during the reverse transition with no EW [56]. 
The second factor inhibiting droplet reversibility arises from various nonconservative dissipative forces which oppose fluid motion and which cannot be directly accounted for in energy minimizationbased modeling frameworks. The primary dissipative forces opposing EW-induced droplet motion on a smooth surface have been studied by various researchers; Bahadur and Garimella [26] showed contact-line friction and wall shear stress to be the dominant dissipative forces. For the case of droplets in the Wenzel state, contact-line pinning at the pillar edges also offers substantial resistance to fluid motion in addition to contact-line friction and viscous forces. The physics underlying contact line pinning are not well understood, which makes it difficult to incorporate pinning in droplet flow models; there have been recent efforts to incorporate contact line pinning effects on smooth [48] and structured surfaces $[79,80]$.

Due to the complexities involved in modeling the flow inside a droplet in the Wenzel state, most studies have analyzed reversibility through experimentation. However, before discussing experimental results it is important to clearly outline the observable criteria which serve as indicators of reversibility. Two distinct and independent criteria for the study of reversibility were introduced by Bahadur and Garimella [56]. The first consists of the extent to which the contact angle retracts upon removal of the EW voltage, while the second is an examination of whether the original Cassie state is attained upon removal of the EW voltage. Both these criteria must be independently satisfied to conclude complete reversibility of the transition. It is important to note that complete contact angle retraction upon removal of the EW voltage does not in itself indicate complete transition reversibility. To illustrate this point, the predicted Cassie and Wenzel angles for a surface with $r_{m}=1.91$ and $\phi=0.41$ are $140^{\circ}$ and $144^{\circ}$, respectively; measurement of contact angle retraction is therefore an insufficient indicator of reversibility since the contact angle values are similar in the non-EW Cassie and Wenzel states. An additional examination of the retraction to the Cassie state is therefore essential to quantify the extent of transition reversibility.

Krupenkin et al. [71] and Herbertson et al. [68] did not observe a reverse transition to the Cassie state upon removal of the EW voltage. Verplanck et al. [77] obtained complete contact angle retraction of a saline droplet on silicon nanowire surfaces in air and undecane environments; however no independent measurements were done to confirm the existence of the Cassie state upon removal of the EW voltage. Bahadur and Garimella [56] carried out a detailed investigation of transition reversibility by quantifying the spontaneous reversibility on eight microstructured surfaces; no retraction of the droplet to the Cassie state (upon removal of the EW voltage) was observed. The contact angle retracted upon the removal of the EW voltage and the extent of the retraction was seen to depend significantly on the surface roughness. Figure 8 shows droplet images on two different surfaces showing contrasting tendencies with respect to contact angle retraction. Figures $8 \mathrm{a}-\mathrm{c}$ show images for a surface which has a roughness $r_{m}$ of 1.36; for this surface, the droplet is in the Wenzel state even in the absence of an EW voltage. It can be seen that a high degree of contact angle retraction is observed on this surface upon the removal of the EW voltage. Figures 8d-f show images for a surface with roughness $r_{m}$ of 3.71 ; for this surface, the droplet is in the Cassie state in the absence of an EW voltage. It can be seen that this surface shows poor contact angle retraction upon removal of the EW voltage. The results show a clear dependence of the contact angle retraction on the surface roughness; higher roughness for the same value of $\phi$ led to a decrease in the contact angle retraction. The results of Bahadur and Garimella [56] highlight the importance of dissipative forces in influencing reversibility.

There are two available experimental results in the existing literature which conclusively demonstrate the Wenzel-Cassie transition. The first demonstration of complete reversibility was by Krupenkin et al. [72] in which an electrically generated heat pulse through the pillared substrate (while the droplet was in the Wenzel state) vaporized a part of the liquid in immediate contact with the pillars. The creation of a thin vapor film at the solid-liquid interface eliminates the dissipative forces inhibiting reversibility; consequently the droplet reverts to the energetically preferred Cassie state. This reversibility was directly visualized by Krupenkin et al. [72] using high speed imaging. However the elevated temperatures resulting from the heat pulse lead to mass loss in the droplet due to evaporation. The second demonstration of complete reversibility was by Dhindsa et al. [76] and consisted of a 


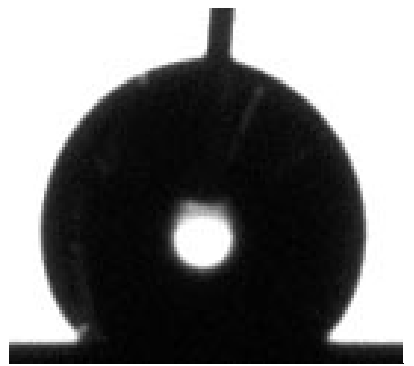

(a) $0 \mathrm{~V}$

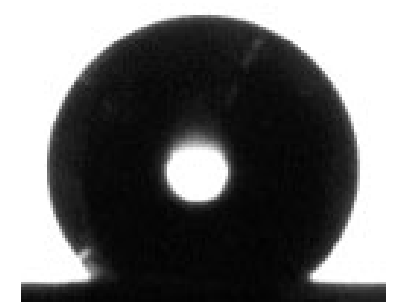

(d) $0 \mathrm{~V}$

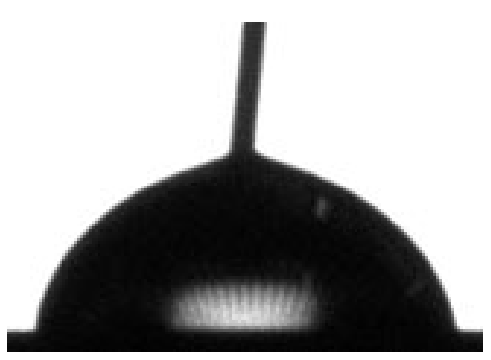

(b) $100 \mathrm{~V}$

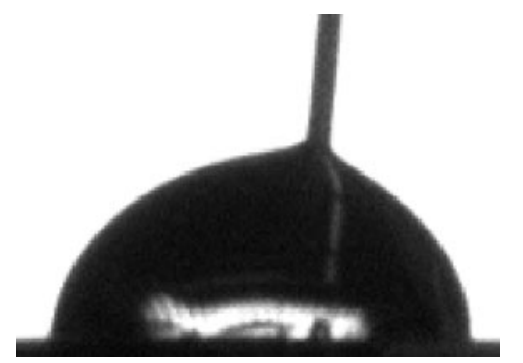

(e) $100 \mathrm{~V}$

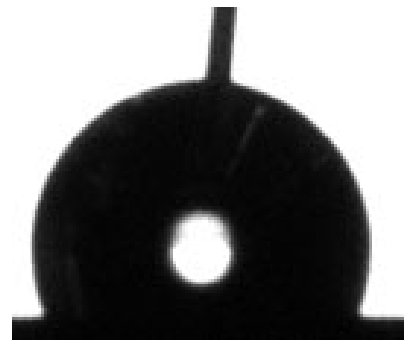

(c) $0 \mathrm{~V}$

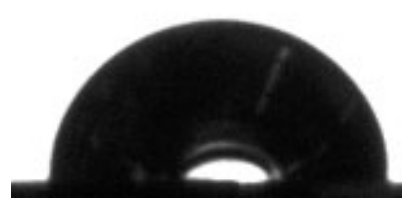

(f) $0 \mathrm{~V}$

Figure 8. Contact angle retraction on microstructured surfaces: (a)-(c) droplet on surface with $r_{m}=1.36$ showing significant contact angle retraction (64\%) after removal of the EW voltage, and (d)-(f) droplet on surface with $r_{m}=3.71$ showing poor contact angle retraction (16\%) [56].

reversible transition of a saline droplet on a vertically aligned carbon nanofiber film. A saline droplet in an air environment did not exhibit any reversibility; however experiments on a saline droplet immersed in a dodecane oil bath showed complete reversibility upon the removal of the EW voltage. Dodecane occupied the space under the saline droplet in the Cassie state and was expelled during the EW-induced Cassie-Wenzel transition. Turning off the voltage resulted in dodecane expelling water between the carbon nanofibers; consequently the droplet reverted to the Cassie state. Complete reversibility was verified in these measurements by contact angle visualization and by measuring the system capacitance before the Wenzel transition and after the reverse transition to the Cassie state. It should be noted that both the demonstrations of complete reversibility involved the use of external stimuli to enable the Wenzel-Cassie transition; Krupenkin et al. [72] used a heat pulse to revert to the Cassie state while Dhindsa et al. [76] utilized competitive wetting interactions between two different liquids.

\subsection{Influence of roughness elements on the Cassie-Wenzel transition}

The nature of the roughness elements is another parameter which can be utilized for enhanced control of droplet states and transitions. Available studies of EW-induced control on structured surfaces have utilized surfaces with roughness elements in the microscale $[18,56,68-70]$ and the nanoscale [71-78] regime. There is no available study on the influence of the size of the roughness elements on the CassieWenzel transition or subsequent spreading in the Wenzel state. It is expected that nanostructured roughness elements will offer larger resistance to liquid motion inside the structured substrate than microstructured elements; however, this difference has not been experimentally quantified. The shape of the roughness elements is also expected to influence phenomena such as contact line pinning and viscous stress which influence droplet behavior in the Wenzel state. The strength and influence of contact line pinning is expected to be significantly lower for surfaces consisting of circular pillars instead of rectangular pillars due to the absence of sharp corners in the former. 


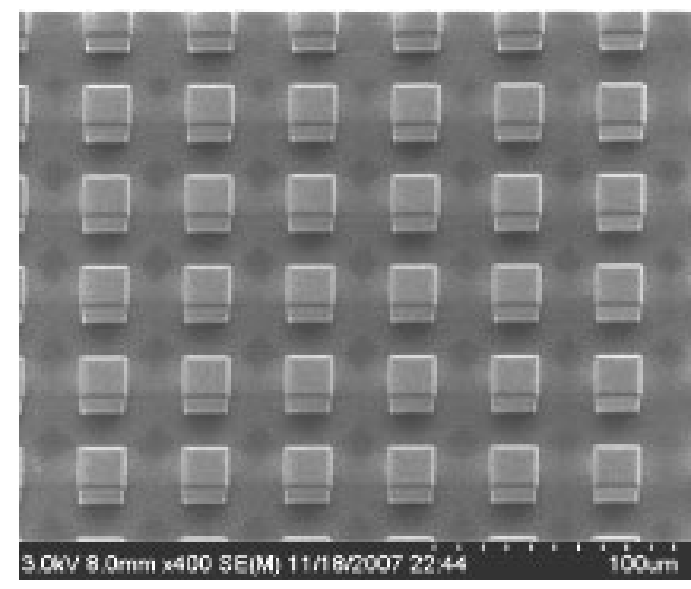

(a)

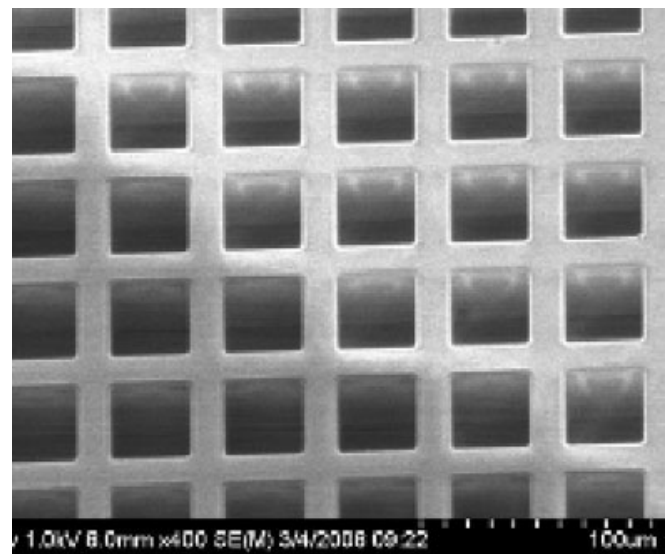

(b)

Figure 9. Structured surfaces with (a) communicating (pillared), and (b) non-communicating (cratered) roughness elements [70].

A majority of the existing studies on EW-induced droplet state control have utilized surfaces in which the medium between the roughness elements is continuous and able to communicate across the elements. Pillared surfaces (Figure 9a) are examples of surfaces in which the medium between the roughness elements is continuous and connected. Consequently, this medium is expelled out of the substrate when the droplet transitions to the Wenzel state. Bahadur and Garimella [70] showed that the nature of droplet transition is fundamentally different on surfaces in which the medium is confined to remain within the roughness elements and cannot escape from the sides. Such a situation occurs when the roughness elements are non-communicating in nature (Figure 9b). On such surfaces the medium is trapped below the droplet (in the Cassie state) and the non-communicating element. In order to attain the Wenzel state, the droplet must expel this medium. Bahadur and Garimella [70] showed that this expulsion is not always possible. Consequently surfaces with non-communicating elements present a higher resistance to the Cassie-Wenzel transition than those with communicating elements.

This concept of utilizing surfaces with non-communicating roughness elements to enhance the robustness of the Cassie state was developed and studied by Bahadur and Garimella [70]. The studies of Krupenkin et al. [72] ('nanobrick' structures) and Ahuja et al. [78] ('honeycomb' structures) also utilized surfaces with noncommunicating roughness elements; however no attempt to quantify the increased robustness of the Cassie state on such surfaces was reported. Bahadur and Garimella [70] conducted detailed experiments to quantify the increase in resistance offered by non-communicating cratered surfaces (Fig 9b) over equivalent communicating pillared surfaces (Fig 9a). On the cratered surfaces, the measured Cassie-Wenzel transition voltages were three times higher than those predicted by energy minimization-based models; on equivalent pillared surfaces, the measured transition voltages agreed reasonably well with predictions from energy minimization-based models. These results indicate that cratered surfaces significantly increase the resistance to the Wenzel transition and enhance the robustness of the Cassie state; this reduces the possibility of an unwanted Wenzel transition triggered by stray energy inputs. Bahadur and Garimella [70] also identified the presence of trapped air (inside the craters) and the resistance to fluid motion by the crater boundaries as the two primary causes for the increased resistance to the Wenzel transition.

Bahadur and Garimella [70] also discovered that the trapped air is not expelled from underneath the droplet (on cratered surfaces) even when the droplet transitions to the Wenzel state. Utilizing optical microscopy, it was shown that the droplet in the Wenzel state only wets the craters that lie beneath the droplet periphery in the initial Cassie state, from where the air can be expelled from the sides; this is illustrated schematically in Figure 10. The air trapped in the craters that are completely covered by the 
droplet base (inside the perimeter of the droplet), on the other hand, cannot escape and thus remains trapped beneath the droplet surface in the Wenzel state. The EW-induced transition on cratered surfaces thus results in a hybrid droplet state in which the peripheral regions of the droplet are in a 'Wenzel' state whereas the central regions are in a 'Cassie' state.

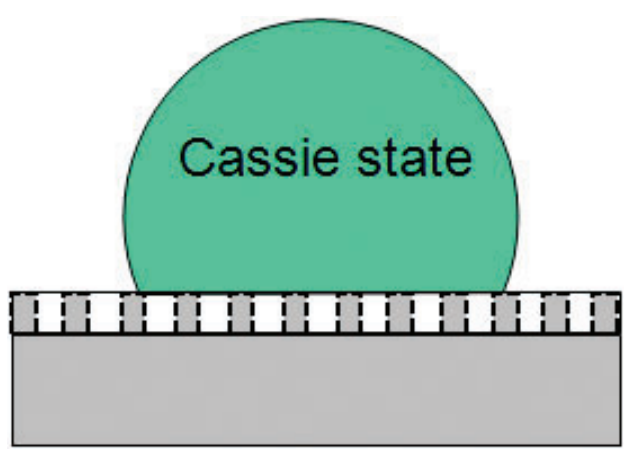

(a)

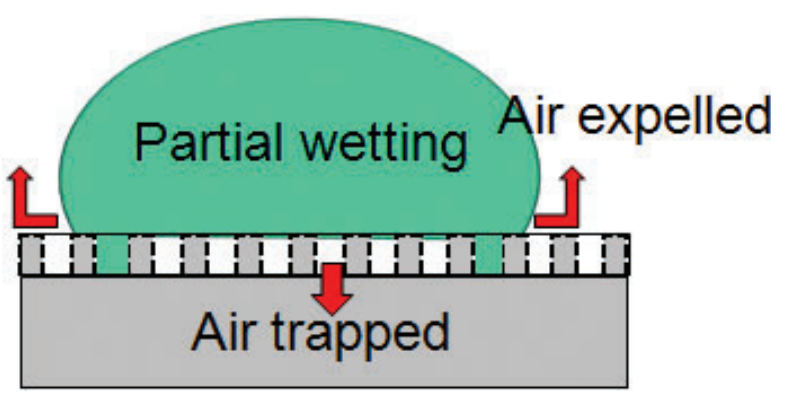

(b)

Figure 10 . Schematic of a droplet on cratered surfaces in (a) Cassie state, and (b) a state where the peripheral regions of the droplet wet the surface but the central regions have an air gap underneath the droplet surface.

\subsection{Applications in heat transfer}

This section discusses potential heat transfer applications of superhydrophobic surfaces; applications of such surfaces in other areas have been extensively analyzed in the available literature and are not considered here. The heat dissipation associated with a moving droplet has promising applications in the area of microelectronics thermal management. A number of researchers [24, 27, 50, 69] have conducted preliminary heat transfer experiments and analyses; the results suggest that droplet-based systems offer significant opportunities for hot-spot and chip-level thermal management. The use of robust superhydrophobic surfaces in such applications offers the additional advantages of a reduction in operating voltages, reduced preheating of the droplet and enhanced flow control. Furthermore, the Cassie and Wenzel states offer contrasting resistances to heat flow from the substrate to the droplet. Dynamic electrical control of these states forms the basis of a thermal resistance switch as conceptualized by Bahadur and Garimella [69], which has potential applications in site-specific thermal management.

Appropriate surface structuring in conjunction with electrowetting can also enable enhanced control of condensation. There is very limited and recent research [81, 82] on condensation patterns on superhydrophobic surfaces. Superhydrophobic surfaces which promote dropwise condensation in the Cassie state will facilitate condensate purging, thereby lowering the thermal resistance to heat transfer; condensate purging can also be enhanced through EW-based pumping of the condensed droplets. This technology has important applications in the field of condensers and heat exchangers, wherein the heat transfer rate can be significantly increased through optimized surface design. Additional applications include the development of anti-dew coatings and water-harvesting coatings [83]. The development of all these applications requires a careful and controlled study of the influence of surface morphology and electric fields on droplet condensation patterns.

\subsection{Avenues for future research}

The fundamental physics underlying EW-induced control of static droplet states and transitions on structured surfaces was discussed above. It is seen that energy minimization-based modeling constitutes an appropriate starting point for the analysis of various aspects of the Cassie and Wenzel states, and of the transition between these states. However, several nonconservative effects coming into play cannot 
be directly incorporated in energy minimization-based frameworks. Effects such as droplet pinning and contact angle hysteresis can play a more dominant role in influencing droplet morphology than surface forces. Current understanding of these phenomena is limited and often relies on semi-empirical data. Energy minimization-based models also do not account for the dynamics of the Cassie-Wenzel transition; it has been shown that the fluid dynamics can exert significant influence on the final nature of transition. A concerted research effort is needed to understand the nature and magnitude of these nonconservative force fields in order to engineer surfaces which minimize their effects.

To date, a complete spontaneous reversibility of the Cassie-Wenzel transition has not been demonstrated. This has severely limited the use of superhydrophobic surfaces in microfluidic applications despite the significant benefits associated with the use of such low-friction surfaces. The main factors impeding reversibility are the energy barrier to the reverse transition and dissipative frictional forces. The key to achieving reversibility is the reduction/elimination of the energy barrier, and an active energy input to trigger the reverse transition and overcome dissipative forces. The role of the shape and length scales of the roughness elements has also been largely unexplored. Recent progress in nanofabrication has significantly increased opportunities for the creation of complex surface morphologies through control of the nature, size, shape, material and hierarchy of the roughness elements; this opens up unexplored tools for enhanced control of droplet states.

Recent research [84] has shown that low surface tension (highly wetting) liquids can also attain Cassie states with contact angles greater than $150^{\circ}$ on structured surfaces with reentrant surface features. Extensive further research is required for the development of technologies to electrically manipulate droplet states and motion of a generic liquid droplet on such omniphobic surfaces. Fundamental research in all of these areas will enable the development of a new class of microfluidic superomniphobic devices with enhanced functionality, higher liquid throughput, low power consumption and increased reliability.

\section{REFERENCES}

[1] T. S. Sammarco and M. A. Burns, Thermocapillary pumping of discrete drops in microfabricated analysis devices, AIChE Journal, 45, 1999, 350-366.

[2] P. Y. Chiou, H. Moon, H. Toshiyoshi, C. J. Kim and M. C. Wu, Light actuation of liquid by optoelectrowetting, Sensors and Actuators A, 104, 2003, 222-228.

[3] X. Geng, H. Yuan, H. N. Oguz and A. Prosperetti, Bubble-based micropump for electrically conducting liquids, Journal of Micromechanics and Microengineering, 11, 2001, 270-276.

[4] F. Mugele and J. C. Baret, Electrowetting: from basics to applications, Journal of Physics.: Condensed Matter, 17, 2005, R705-R774.

[5] R. B. Fair, Digital microfluidics: is a true lab-on-a-chip possible?, Microfluidics and Nanofluidics, 3, 2007, 245-281.

[6] S. Y. Teh, R. Lin, L. H. Hung and A. P. Lee, Dropelt microfluidics, Lab on a Chip, 8, 2008, 198220.

[7] A. Quinn, R. Sedev and J. Ralston, Contact angle saturation in electrowetting, Journal of Physical Chemistry B, 109, 2005, 6268-6275.

[8] M. Vallet, M. Vallade and B. Berge Limiting phenomena for the spreading of water on polymer films by electrowetting, European Physical Journal B, 11, 1999, 583-591.

[9] A. Quinn, R. Sedev J. Ralston, Influence of the electrical double layer in electrowetting, Journal of Physical Chemistry B, 107, 2003, 1163-1169.

[10] H. J. J. Verheijen and M. W. J. Prins, Reversible electrowetting and trapping of charge: model and experiments, Langmuir, 15, 1999, 6616-6620.

[11] T. B. Jones, An electromechanical interpretation of electrowetting, Journal of Micromechanics and Microengineering, 15, 2005, 1184-1187. 
[12] N. Kumari, V. Bahadur and S. V. Garimella, Electrical actuation of electrically conducting and insulating droplets using ac and dc voltages, Journal of Micromechanics and Microengineering, $18,2008,105015$.

[13] D. Quere, Non-sticking drops, Reports on Progress in Physics, 68, 2005, 2495-2532.

[14] X. IL, D. Reinhoudt and M. Crego-Calama, What do we need for a superhydrophobic surface? A review on the recent progress in the preparation of superhydrophobic surfaces, Chemical Society Reviews, 36, 2007, 1350-1368.

[15] M. Nosonovsky and B. Bhushan, Superhydrophobic surfaces and emerging applications: nonadhesion, energy, green engineering, Current Opinion in Interface and Colloid Science, 2009.

[16] X. Zhang, F. Shi, J. Niu, Y. Jiang and Z. Wang, Superhydrophobic surfaces: from structural control to functional application, Journal of Materials Chemistry, 18, 2008, 621-633.

[17] J. Heikenfeld and M. Dhindsa, Electrowetting on superhydrophobic surfaces: Present status and prospects, Journal of Adhesion Science and Technology, 22, 2008, 319-334.

[18] V. Bahadur and S. V. Garimella, Electrowetting-based control of static droplet states on rough surfaces, Langmuir, 23, 2007, 4918-4924.

[19] C. J. Kim, Micropumping by electrowetting, ASME International Mechanical Engineering Congress and Exposition New York IMECE2001/HTD-24200, 2001.

[20] M. G. Pollack, A. D. Shenderov and R. B. Fair, Electrowetting-based actuation of droplets for integrated microfluidics, Lab on a Chip, 2, 2002, 96-101.

[21] M. G. Pollack and R. B. Fair, Electrowetting-based actuation of liquid droplets for microfluidic applications, Applied Physics Letters, 77, 2000, 1725-1726.

[22] K. Mohseni and A. Dolatabadi, Electrowetting droplet actuation in micro scale devices, 43rd AIAA Aerospace Science Meeting and Exhibit, Nevada AIAA-677, 2005.

[23] J. S. Kuo, P. Spicar-Mihalic, I. Rodriguez and D. T. Chiu, Electrowetting-induced droplet movement in an immiscible medium, Langmuir, 19, 2003, 250-255.

[24] P. Paik, V. K. Pamula and K. Chakrabarty, Adaptive hot-spot cooling of integrated circuits using digital microfluidics, Proceedings of IMECE 2005 IMECE2005-81081, Florida (USA), 2005.

[25] R. B. Fair, V. Srinivasan, H. Ren, P. Paik, V. K. Pamula and M. G. Pollack, Electrowetting-based on-chip sample processing for integrated microfluidics, IEEE International Electron Devices Meeting, 2003.

[26] V. Bahadur and S. V. Garimella, An energy-based model for electrowetting-induced droplet actuation, Journal of Micromechanics and Microengineering, 16, 2006, 1494-1503.

[27] H. Oprins, B. Vandevelde, E. Beyne, G. Borghs and M. Baelmans, Selective cooling of microelectronics using electrostatic actuated liquid droplets-modeling and experiments, International Workshop on Thermal Investigations of ICs and Systems, Cote d'Azur (France), 2004, 207-212.

[28] L. Lee, H. Moon, B. Fowler , T. Schoellhammer and C. J. Kim, Electrowetting and electrowetting-on-dielectric for microscale liquid handling, Sensors and Actuators A, 95, 2002, 259-268.

[29] D. Chatterjee, B. Hetayothin, A. R. Wheeler, D. J. King and R. L. Garrell, Droplet-based microfluidics with nonaqueous solvents and solutions, Lab on a Chip, 6, 2006, 199-206.

[30] C. G. Cooney, C. Y. Chen, M. R. Emerling, A. Nadim and J. D. Sterling, Electrowetting droplet microfluidics on a single planar surface, Microfluidics and Nanofluidics, 2, 2006, 435-446.

[31] U. C. Yi and C. J. Kim, Characterization of electrowetting actuation on addressable single-side coplanar electrodes, Journal of Micromechanics and Microengineering, 16, 2006, 2053-2059.

[32] A. Torkkeli, J. Saarilahti, A. Haara, H. Harma, T. Soukka and P. Tolonen, Electrostatic transportation of water droplets on superhydrophobic surfaces, 14th International IEEE Conference on MEMS, 2001, 475-478. 
[33] A. Kumar, M. Pluntke, B. Cross, J. C. Baret and F. Mugele, Finite conductivity effects and apparent contact angle saturation in AC electrowetting, Materials Research Society Symposium Proceedings 899E 0899-N06-01.1-8, 2006.

[34] T. B. Jones, Liquid dielectrophoresis on the microscale, Journal of Electrostatics, 51, 2001, 290299.

[35] R. Baviere, J. Boutet and Y. Fouilliet, Dynamics of droplet transport by electrowetting actuation, Microfluidics and Nanofluidics, 4, 2008, 287-294.

[36] M. Washizu, Electrostatic actuation of liquid droplets for micro-reactor applications, IEEE Industry Applications Society Annual Meeting, New Orleans, USA, 1997.

[37] B. Berge and J. Peseux, Variable focal lens controlled by an external voltage: an application of electrowetting, European Physical Journal E, 3, 2000, 159-163.

[38] N. Kumari, V. Bahadur and S. V. Garimella, Electrical actuation of dielectric droplets, Journal of Micromechanics and Microengineering, 18, 2008, 085018.

[39] T. Ward, Electrohydrostatic wetting of poorly-conducting liquids, Journal of Electrostatics, 64 , 2006, 817-825.

[40] H. Pellat, Mesure de la force agissant sur les diélectriques liquides non électrisés placés dans un champ élitrique, C. Royal Academy Science Paris, 119, 1895, 691-694.

[41] T. B. Jones, M. Gunji, M. Washizu and M. J. Feldman, Dielectrophoretic liquid actuation and nanodroplet formation, Journal of Applied Physics, 89, 2001, 1441-1448.

[42] T. B. Jones, K. L. Wang and D. J. Yao, Frequency dependent electromechanics of aqueous liquids: Electrowetting and dielectrophoresis, Langmuir, 20, 2004, 2813-2818.

[43] T. B. Jones, J. D. Fowler, Y. S. Chang and C. J. Kim, Frequency-based relationship of electrowetting and dielectrophoretic liquid microactuation, Langmuir, 19, 2003, 7646-7651.

[44] J. H. Song, R. Evans, Y. Y. Lin, B. N. Hsu and R. B. Fair, A scaling model for electrowetting-ondielectric microfluidic actuators, Microfluidics and Nanofluidics, 2009.

[45] E. Baird, P. Young and K. Mohseni, Electrostatic force calculation for an EWOD-actuated droplet, Microfluidics and Nanofluidics, 3, 2007, 635-644.

[46] T. B. Jones, More about the electromechanics of electrowetting, Mechanics Research Communications, 36, 2009, 2-9.

[47] D. Chatterjee, H. Shepherd and R. L. Garrell, Electromechanical model for actuating liquids in a two-plate droplet microfluidic device, Lab on a Chip , 9, 2009, 1219-1229.

[48] S. W Walker and B. Shaipro, Modeling the fluid dynamics of electrowetting on dielectric (EWOD), Journal of Microelectromechanical Systems, 15, 2006, 986-1000.

[49] T. D. Blake, Dynamic contact angles and wetting kinetics in Wettability, Surfactant Science Series 49, Marcel Dekker, New York, 1993, 251-309.

[50] K. Mohseni, E. Baird and H. Zhao, Digitized heat transfer for thermal management of compact systems, Proceedings of the ASME International Mechanical Engineering Congress and Exposition, IMECE2005-79372, Orlando, USA, 2005.

[51] M. Schneemilch, W. J. J. Welters, R. A. Hayes and J. Ralston, Electrically induced changes in dynamic wettability, Langmuir, 16, 2000, 2924-2927.

[52] H. Lu, F. Bottausci, J. D. Fowler, A. L. Bertozzi, C. Meinhart and C. J. Kim, A study of EWODdriven droplets by PIV investigation, Lab on a Chip, 8, 2008, 456-461.

[53] H. Oprins, J. Danneels, B. V. Ham, B. Vandevelde and M. Baelmans, Convection heat transfer in electrostatic actuated liquid droplets for electronics cooling, Proceedings of Thermal Challenges in Next Generation Electronic Systems, Santa Fe, New Mexico, USA, 2007, 233-230.

[54] H. Ren, R. B. Fair, M. G. Pollack and E. J. Shaughnessy, Dynamics of electro-wetting droplet transport, Sensors and Actuators B, 87, 2002, 201-206. 
[55] B. Barbier, C. Combettes, S. Guillemet-Fritsch, T. Chartier, F. Rossignol, A. Rumeau, T. Lebey and E. Dutarde, CaCu3Ti4O12 ceramics from co-precipitation method : Dielectric properties of pellets and thick films, Journal of the European Ceramic Society, 29, 2009, 731-735.

[56] V. Bahadur and S. V. Garimella, Electrowetting-based control of droplet transition and morphology on artificially microstructured surfaces, Langmuir, 24, 2008, 8338-8345.

[57] A. B. D. Cassie, Contact Angles, Discussions of the Faraday Society, 3, 1948, 11-16.

[58] T. N. Wenzel, Surface roughness and contact angle, Journal of Physical Colloid Chemistry, 53, 1949, 1466-1467.

[59] N. A. Patankar, Mimicking the lotus effect: Influence of double roughness structures and slender pillars, Langmuir, 20, 2004, 8209-8213.

[60] Y. Yu, Z. Zhao and Q. Zheng, Mechanical and superhydrophobic stabilities of two-scale surfacial structure of lotus leaves, Langmuir, 23, 2007, 8212-8216.

[61] A. Marmur, The lotus effect: superhydrophobicity and metastability, Langmuir, 20, 2004, 35173519.

[62] N. A. Patankar, On the modeling of hydrophobic contact angles on rough surfaces, Langmuir, 19, 2003, 1249-1253.

[63] B. He, N. A. Patankar and J. Lee, Multiple equilibrium droplet shapes and design criterion for rough hydrophobic surfaces, Langmuir, 19, 2003, 4999-5003.

[64] N. A. Patankar, Transition between superhydrophobic states on rough surfaces, Langmuir, 20, 2004, 7097-7102.

[65] B. Liu, and F. F. J. Lange, Pressure induced transition between superhydrophobic states: configuration diagrams and effect of surface feature size, Journal of Colloid and Interface Science, 298, 2006, 899-909.

[66] M. Nosonovsky and B. Bhushan, Patterned nonadhesive surfaces: superhydrophobicity and wetting regime transitions, Langmuir, 24, 2008, 1525-1533.

[67] K. Varanasi, T. Deng, M. F. Hsu and N. Bhate, Design of superhydrophobic surfaces for optimum roll-off and droplet impact resistance, Proceedings of the ASME International Mechanical Engineering Congress and Exposition, IMECE2008-67808, Boston, USA, 2008.

[68] D. J. Herbertson, R. E. Evans, N. J. Shirtcliffe, G. McHale and M. I. Newton, Electrowetting on superhydrophobic SU-8 patterned surfaces, Sensors and Actuators A, 130, 2006, 189-193.

[69] V. Bahadur and S. V. Garimella, Energy minimization-based analysis of electrowetting for microelectronics cooling applications, Microelectronics Journal, 39, 2008, 957-965.

[70] V. Bahadur and S. V. Garimella, Preventing the Cassie-Wenzel transition using surfaces with noncommunicating roughness elements, Langmuir, 28, 2009, 4815-4820.

[71] T. N. Krupenkin, J. A. Taylor, T. M. Schneider and S. Yang, From rolling ball to complete wetting: The dynamic tuning of liquids on nanostructured surfaces, Langmuir, 20, 2004, 3824-3827.

[72] T. N. Krupenkin, J. A. Taylor, E. N. Wang, P. Kolodner, M. Hodes, and T. R. Salamon, Reversible wetting-dewetting transitions on electrically tunable superhydrophobic nanostructured surfaces, Langmuir, 23, 2007, 9128-9133.

[73] L. Zhu, J. Xu, Y. Xiu, Y. Sun, D. W. Hess and C. Wong, Electrowetting of aligned carbon nanotube films, Journal of Physical Chemistry B, 110, 2006, 15945-15950.

[74] Z. Wang, Y. Ou, T. Lu and N. Koratkar, Wetting and electrowetting properties of carbon nanotube templated parylene films, Journal of Physical Chemistry B, 111, 2007, 4296-4299.

[75] Z. Wang, L. Ci, L. Chen, S. Nayak, P. Ajayan and N. Koratkar, Polarity-dependent electrochemically controlled transport of water through carbon nanotube membranes, Nanoletters, 7, 2007, 697-703. 
[76] M. Dhindsa, N. R. Smith, J. Heikenfeld, P. D. Rack, J. D. Fowlkes, M. J. Doktycz, A. V. Melechko and M. L. Simpson, Reversible electrowetting of vertically aligned superhydrophobic carbon nanofibers, Langmuir, 22, 2006, 9030-9034.

[77] N. Verplanck, E. Galopin, J. Camart, V. Thomy, Y. Coffinier and R. Boukherroub, Reversible electrowetting on superhydrophobic silicon nanowires, Nanoletters, 7, 2007, 813-817.

[78] A. Ahuja, J. A. Taylor, V. Lifton, A. A. Sidorenko, T. R. Salamon, E. J. Lobaton, P. Kolodner and T. N. Krupenkin, Nanonails: A simple geometric approach to electrically tunable superlyophobic surfaces, Langmuir, 24, 2008, 9-14.

[79] C. Dorrer and J. Ruhe, Drops on microstructured surfaces coated with hydrophilic polymers: Wenzel's model and beyond, Langmuir, 24, 2008, 1959-1964.

[80] C. Priest, T. W. J. Albrecht, R. Sedev and J. Ralston, Asymmetric wetting hysteresis on hydrophobic microstructured surfaces, Langmuir, 25, 2009, 5655-5660.

[81] C. Dorrer and J. Ruhe, Condensation and wetting transitions on microstructured ultrahydrophobic surfaces, Langmuir, 23, 2007, 3820-3824.

[82] C. H. Chen, Q. Cai, C. Tsai, C. L. Chen, G. Xiong, Y. Yu and Z. Ren, Dropwise condensation on superhydrophobic surfaces with two-tier roughness, Applied Physics Letters, 90, 2007, 173108.

[83] R. P. Garrod, L. G. Harris, W. C. E. Schofield, J. McGettrick, L. J. Ward, D. O. H. Teare and J. P. S. Badyal, Mimicking a Stenocara Beetle's back for microcondensation using plasmachemical patterned superhydrophobic-superhydrophilic surfaces, Langmuir, 23, 2007, 689-693.

[84] A. Tuteja, W. Choi, J. M. Mabry, G. H. McKinley and R. E. Cohen, Robust omniphobic surfaces, Proceedings of the National Academy of Sciences of the United States of America, 105, 2008, 18200-18205. 